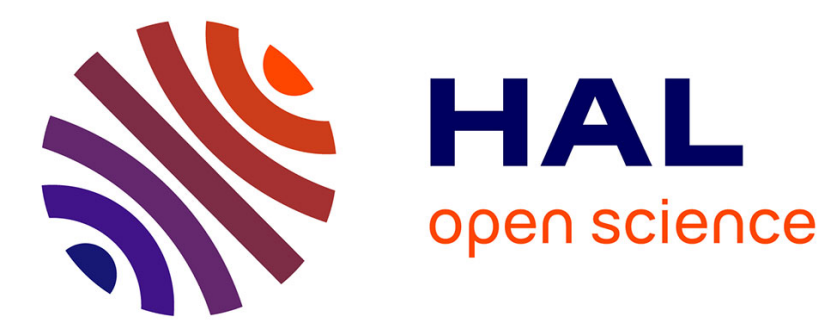

\title{
Widening the scope of an eigenvector stochastic approximation process and application to streaming PCA and related methods
}

\author{
Jean-Marie Monnez, Abderrahman Skiredj
}

\section{To cite this version:}

Jean-Marie Monnez, Abderrahman Skiredj. Widening the scope of an eigenvector stochastic approximation process and application to streaming PCA and related methods. Journal of Multivariate Analysis, 2021, 182, pp.104694. 10.1016/j.jmva.2020.104694 . hal-03038206

\section{HAL Id: hal-03038206 \\ https://hal.inria.fr/hal-03038206}

Submitted on 3 Dec 2020

HAL is a multi-disciplinary open access archive for the deposit and dissemination of scientific research documents, whether they are published or not. The documents may come from teaching and research institutions in France or abroad, or from public or private research centers.
L'archive ouverte pluridisciplinaire HAL, est destinée au dépôt et à la diffusion de documents scientifiques de niveau recherche, publiés ou non, émanant des établissements d'enseignement et de recherche français ou étrangers, des laboratoires publics ou privés. 


\title{
Widening the Scope of an Eigenvector Stochastic Approximation Process and Application to Streaming PCA and Related Methods
}

\author{
Jean-Marie Monnez ${ }^{*, a}$, Abderrahman Skiredj **,b \\ ${ }^{a}$ Université de Lorraine, CNRS, Inria ${ }^{1}$, IECL ${ }^{2}$, F-54000 Nancy, France E CHRU Nancy, INSERM, CIC-P ${ }^{3}$, F-54000 Nancy, France \\ ${ }^{b}$ Université de Lorraine, CNRS, IECL, F-54000 Nancy, France E Ecole Nationale Supérieure des Mines de Nancy ${ }^{4}, F-54000$ Nancy, France
}

\begin{abstract}
We prove the almost sure convergence of Oja-type processes to eigenvectors of the expectation $B$ of a random matrix while relaxing the i.i.d. assumption on the observed random matrices $\left(B_{n}\right)$ and assuming either $\left(B_{n}\right)$ converges to $B$ or $\left(E\left[B_{n} \mid T_{n}\right]\right)$ converges to $B$ where $T_{n}$ is the sigma-field generated by the events before time $\mathrm{n}$. As an application of this generalization, the online PCA of a random vector $Z$ can be performed when there is a data stream of i.i.d. observations of $Z$, even when both the metric $M$ used and the expectation of $Z$ are unknown and estimated online. Moreover, in order to update the stochastic approximation process at each step, we are no longer bound to using only a mini-batch of observations of $Z$, but all previous observations up to the current step can be used without having to store them. This is useful not only when dealing with streaming data but also with Big Data as one can process the latter sequentially as a data stream. In addition the general framework of this process, unlike other algorithms in the literature, also covers the case of factorial methods related to PCA.
\end{abstract}

AMS 2020 subject classifications:

Primary: 62L20

Secondary: 60L10, 62H 25

Keywords: Big Data, Data Stream, Eigenvectors, Online Estimation, Principal Component Analysis, Stochastic Algorithms, Stochastic Approximation.

\section{Introduction}

Streaming data are data that arrive continuously such as process control data, web data, telecommunication data, medical data, financial data, etc. In this setting, recursive stochastic algorithms can be used to estimate online, among others, parameters of a regression function (for example, see [12] and references therein) or centers of clusters in unsupervised classification [8] or principal components in a principal component analysis (PCA) (for example, see [13], p. 343). More precisely, each arriving observation vector is used to update the estimate sequence until it converges to the quantity of interest. When using such processes, it is not necessary to store the data and, due to the relative simplicity of the computation involved, much more data can be taken into account during the same duration of time than with non sequential methods. Moreover, this type of method uses less memory space than a batch method [1]. In this article, we propose a general framework of stochastic approximation processes and subsequently apply the latter to the case of streaming PCA. This general framework is sufficiently flexible to cover the case of streaming normed PCA and other related methods while allowing the stochastic algorithm to absorb a greater amount of data at each step or even all of the previously observed data up to the current step without any additional memory storage. This can be extremely efficient for dealing with large data streams.

\footnotetext{
${ }^{1}$ Inria, project-team BIGS

${ }^{2}$ Institut Elie Cartan de Lorraine, BP 239, F-54506 Vandœuvre-lès-Nancy, France

${ }^{3}$ Centre d'Investigation Clinique Plurithématique

${ }^{4}$ Campus Artem, CS 14234, 92 Rue Sergent Blandan, F-54042 Nancy, France

**Corresponding author.*jean-marie.monnez@univ-lorraine.fr**abderrahman.skiredj@mines-nancy.org
} 
Let us define some notations. Let $A^{\top}$ denote the transpose of a matrix $A$. Let $Q$ be a positive definite symmetric $(p, p)$ matrix called metric, $\langle.,$.$\rangle and \|$.$\| be respectively the inner product and the norm in \mathbb{R}^{p}$ induced by $Q:\langle x, y\rangle=$ $x^{\top} Q y, x^{\top}$ denoting the transpose of the column vector $x$. For vectors in $\mathbb{R}^{p}, Q$-orthogonal and $Q$-normed respectively mean orthogonal and normed with respect to the metric $Q$. Recall that a $(p, p)$ matrix $A$ is $Q$-symmetric if $(Q A)^{\top}=Q A$; then $A$ has $p$ real eigenvalues and there exists a $Q$-orthonormal basis of $\mathbb{R}^{p}$ consisting of eigenvectors of $A$. The norm of a matrix $A$ is the spectral norm denoted $\|A\|$. The abbreviation a.s. stands for almost surely.

Numerous articles have been devoted to the problem of estimating eigenvectors and corresponding eigenvalues in decreasing order of the expectation $B$ of a random symmetric $(p, p)$ matrix, using an i.i.d. sample of the latter. These include, among others, the algorithms of Benzécri [2], Krasulina [16], Oja [19], Karhunen and Oja [15], Oja and Karhunen [20], Brandière [3-5], Brandière and Duflo [6] and Duflo [13] in the case of PCA. We consider here the commonly used normed process of Oja [15, 20] $\left(X_{n}\right)$, whose rate of convergence is studied in [1], recursively defined by:

$$
X_{n+1}=\frac{\left(I+a_{n} B_{n}\right) X_{n}}{\left\|\left(I+a_{n} B_{n}\right) X_{n}\right\|},
$$

the random matrices $B_{n}$ being mutually independent and a.s. bounded, $E\left[B_{n}\right]=B, a_{n}>0, \sum_{n=1}^{\infty} a_{n}=\infty, \sum_{n=1}^{\infty} a_{n}^{2}<\infty$. A commonly used choice of $a_{n}$ is $\frac{a}{n^{\alpha}}, \frac{1}{2}<\alpha \leq 1$ (for example, see [12]). This process converges a.s. to a normed eigenvector of $B$ corresponding to its largest eigenvalue.

Consider the application presented in Section 3 to PCA of a random vector $Z$ with unknown expectation $E[Z]$ and covariance matrix $C=E\left[(Z-E[Z])(Z-E[Z])^{\top}\right]$. Suppose there is a data stream $\left(Z_{i}, i \geqslant 1\right)$ of i.i.d. observations of $Z$ and that the metric $M$ used to define the distance between two observations of $Z$ is unknown, for example the diagonal matrix of the inverses of variances of the components of $Z$ in normed PCA. To estimate the principal components, we must estimate eigenvectors of the $M^{-1}$-symmetric matrix $B=M C$ (or of the symmetric matrix $M^{\frac{1}{2}} C M^{\frac{1}{2}}$, see Section $3)$. We can estimate online $E[Z]$ and $M$ respectively at step $n$ by $\bar{Z}_{n-1}$, the empirical mean of $\left(Z_{i}, i \leq n-1\right)$, and $M_{n-1}$ depending on $\left(Z_{i}, i \leq n-1\right)$, and define $B_{n}=M_{n-1}\left(Z_{n}-\bar{Z}_{n-1}\right)\left(Z_{n}-\bar{Z}_{n-1}\right)^{\top}$. It is clear that the random matrices $B_{n}$ do not satisfy the assumptions of Oja. In fact, these assumptions would be verified if the expectations and variances of the components of $Z$ were known a priori in the case of normed PCA or other characteristics in other types of PCA. This is the case for example if we have at our disposal a massive data vectors set with computed characteristics and we randomly draw a data vector from this data set at each step ([13], p. 343). Here we suppose that data arrive continuously and are drawn from an unknown distribution. The convergence of the process in such a case is not proven.

The general convergence results are presented in Section 2, the application to streaming PCA and related methods in Section 3, the conclusion in Section 4, and the proofs of all theorems in Section 5.

Let $Q$ be a metric in $\mathbb{R}^{p}, B$ a $Q$-symmetric matrix and, for $n \geqslant 1, T_{n}$ the $\sigma$-field generated by the events before time $n ; X_{1}, B_{1}, \ldots, B_{n-1}$ are $T_{n}$-measurable. We prove the almost sure convergence of the process of Oja assuming that the conditional expectation of $B_{n}$ with respect to $\sigma$-field $T_{n}$ converges almost surely to $B$ as $n$ goes to infinity (Subsection 2.1, Theorem 1, first part). This allows proving the convergence of the process in the case of streaming PCA when a mini-batch of observations of $Z$ is taken into account at each step (Subsection 3.1). A method of Duflo ([13], p. 343 ) is used in the proof (Section 5), but with more general assumptions. The proof of the convergence of processes $\left(X_{n}^{i}\right), i \in\{1, \ldots, r\}, r \leq p$, of the same type, obtained by a Gram-Schmidt orthonormalization with respect to $Q$, to unit eigenvectors of $B$ corresponding to the $r$ largest eigenvalues in decreasing order (Subsection 2.2, Corollary 2, first part), not given in [13], is established in Section 5.

Moreover, we prove the almost sure convergence of the process of Oja, with an entirely different method, in the non-classical case where $B_{n}$ converges almost surely to $B$ (Subsection 2.1, Theorem 1, second part and Subsection 2.2, Corollary 2, second part; proofs in Section 5). This applies to PCA of a random vector $Z$ while allowing the process to be updated at each step by using all previous observations $\left(Z_{i}\right)$ up to the current step without the need to store the latter (Subsection 3.2). Hence, we define a type of processes different from the classical processes that used a mini-batch of observations at each step. The conducted experiments (see the Appendix), show that these processes are generally faster than the classical processes.

Finally, the scope of these processes is further widened to other factorial methods such as multiple factor analysis [21] or generalized canonical correlation analysis [10] (Subsection 3.3). 


\section{Theorem of almost sure convergence}

\subsection{Estimation of an eigenvector corresponding to the largest eigenvalue}

We make the assumptions given below:

(H1a) $B$ is $Q$-symmetric; let $\lambda_{1}, \lambda_{2}, \ldots, \lambda_{p}$, denote its eigenvalues in decreasing order and for $i \in\{1, \ldots, p\}, V_{i}$ a $Q$ - normed eigenvector of $B$ corresponding to $\lambda_{i}$;

(H1b) The largest eigenvalue $\lambda_{1}$ of $B$ is simple;

(H2a) There exists a positive number $b$ such that $\sup _{n}\left\|B_{n}\right\|<b$ a.s.;

(H2b) $B_{n}$ is not $T_{n}$-measurable, $\sum_{1}^{\infty} a_{n} E\left[\left\|E\left[B_{n} \mid T_{n}\right]-B\right\|\right]<\infty$; (H2b') $\sum_{1}^{\infty} a_{n}\left\|B_{n}-B\right\|<\infty$ a.s.;

(H2c) For all $n, I+a_{n} B_{n}$ is invertible;

(H3) $a_{n}>0, \sum_{1}^{\infty} a_{n}=\infty, \sum_{1}^{\infty} a_{n}^{2}<\infty$;

(H4) $X_{1}$ is a random variable independent of the sequence $\left(B_{n}\right)$;

(H4') $X_{1}$ is an absolutely continuous random variable with respect to the Lebesgue measure and independent of the sequence $\left(B_{n}\right)$.

Assumption $\mathrm{H} 2 \mathrm{~b}$ is obviously verified in the classical case $E\left[B_{n} \mid T_{n}\right]=B$ a.s. According to a classical lemma, given that $\sum_{1}^{\infty} a_{n}=\infty, \mathrm{H} 2 \mathrm{~b}$ implies that there exists a subsequence of $\left(E\left[B_{n} \mid T_{n}\right]\right)$ converging a.s. to $B$. Likewise, H2b' implies that there exists a subsequence of $\left(B_{n}\right)$ converging a.s. to $B$. H2c is verified in particular when the eigenvalues of $B_{n}$ are non-negative. Assumption H3 is classical for Robbins-Monro type processes [12]. Note that in the second part of the theorem, since $\omega \in \Omega$ is fixed throughout the proof, $a_{n}$ can be a positive random variable.

Let $U_{n}=\left\langle X_{n}, B X_{n}\right\rangle$ and $W_{n}=\left\langle X_{n}, B_{n} X_{n}\right\rangle$.

Theorem 1. (i) Suppose assumptions Hla,b, H2a,b, H3, H4 hold. Then, $U_{n}$ converges a.s. to one of the eigenvalues of $B$; on $E_{j}=\left\{U_{n} \longrightarrow \lambda_{j}\right\}, X_{n}$ converges a.s. to $V_{j}$ or $-V_{j}, \sum_{n=1}^{\infty} a_{n}\left(\lambda_{j}-U_{n}\right)$ and $\sum_{n=1}^{\infty} a_{n}\left(\lambda_{j}-W_{n}\right)$ converge a.s. Moreover, if $\liminf E\left[\left\langle B_{n} X_{n}, V_{1}\right\rangle^{2} \mid T_{n}\right]>0$ a.s. on $\cup_{j=2}^{p} E_{j}$, then $P\left(E_{1}\right)=1$;

(ii) Suppose assumptions H1a,b, H2b',c, H3, H4' hold. Then $X_{n}$ converges a.s. to $V_{1}$ or $-V_{1}, \sum_{1}^{\infty} a_{n}\left(\lambda_{1}-U_{n}\right)$ and $\sum_{1}^{\infty} a_{n}\left|\lambda_{1}-W_{n}\right|$ converge a.s.

Note that the methods of proofs of the two parts of Theorem 1 provided in Section 5 are entirely different. The first method is that used by Duflo ([13], p. 343) but with more general assumptions; the assumption "on $\cup_{j=2}^{p} E_{j}$, $\lim \inf E\left[\left\langle B_{n} X_{n}, V_{1}\right\rangle^{2} \mid T_{n}\right]>0$ a.s." is used to avoid the traps $\left(X_{n}\right.$ converges to $\left.V_{j}, j \neq 1\right)$ of the stochastic algorithm; it is verified in the case of PCA of a random vector $Z$ under assumptions H6b and H7b given in Corollary 3.

\subsection{Simultaneous estimation of several eigenvectors}

For $i \in\{1, \ldots, r\}, r \leq p$, recursively define the process $\left(X_{n}^{i}\right)$ in $\mathbb{R}^{p}$ such that:

$$
Y_{n+1}^{i}=\left(I+a_{n} B_{n}\right) X_{n}^{i}, T_{n+1}^{i}=Y_{n+1}^{i}-\sum_{j<i}\left\langle Y_{n+1}^{i}, X_{n+1}^{j}\right\rangle X_{n+1}^{j}, X_{n+1}^{i}=\frac{T_{n+1}^{i}}{\left\|T_{n+1}^{i}\right\|} .
$$

The vector $\left(X_{n+1}^{1}, \ldots, X_{n+1}^{r}\right)$ is obtained by Gram-Schmidt orthonormalization of $\left(Y_{n+1}^{1}, \ldots, Y_{n+1}^{r}\right)$. We make the assumptions given below:

(H1b') The eigenvalues of $B$ are distinct;

(H5) For $i \in\{1, \ldots, r\}, X_{1}^{i}$ is a random variable independent of the sequence $\left(B_{n}\right)$;

(H5') For $i \in\{1, \ldots, r\}, X_{1}^{i}$ is an absolutely continuous random variable with respect to the Lebesgue measure and independent of the sequence $\left(B_{n}\right)$.

\section{Corollary 2.}

(i) Suppose assumptions Hla,b', H2a,b, H3, H5 hold. Then, for $i \in\{1, \ldots, r\}, X_{n}^{i}$ converges a.s. to one of the eigenvectors of B; moreover, if on $\cup_{j=i+1}^{p}\left\{X_{n}^{i} \longrightarrow \pm V_{j}\right\}$, $\liminf E\left[\left\langle B_{n} X_{n}^{i}, V_{i}\right\rangle^{2} \mid T_{n}\right]>0$ a.s., then $X_{n}^{i}$ converges a.s. to $V_{i}$ or $-V_{i}, \sum_{1}^{\infty} a_{n}\left|\lambda_{i}-\left\langle X_{n}^{i}, B X_{n}^{i}\right\rangle\right|$ and $\sum_{1}^{\infty} a_{n}\left(\lambda_{i}-\left\langle X_{n}^{i}, B_{n} X_{n}^{i}\right\rangle\right)$ converge a.s.;

(ii) Suppose assumptions Hla,b', H2b',c, H3, H5' hold. Then, for $i \in\{1, \ldots, r\}, X_{n}^{i}$ converges a.s.to $V_{i}$ or $-V_{i}$, $\sum_{1}^{\infty} a_{n}\left|\lambda_{i}-\left\langle X_{n}^{i}, B X_{n}^{i}\right\rangle\right|$ and $\sum_{1}^{\infty} a_{n}\left|\lambda_{i}-\left\langle X_{n}^{i}, B_{n} X_{n}^{i}\right\rangle\right|$ converge a.s.

The proofs of the two parts of Corollary 2 are provided in Section 5. 


\section{Application to streaming PCA and related methods}

In this section, we apply the theoretical results given in Section 2 to the estimation of the principal components of a streaming PCA. Classical convergence results exist for the process (1) or for processes of the same type. In these processes, a mini-batch of data is used at each step and it is assumed that the random matrices $B_{n}$ are independent. However, in practice, the metric $M$ used to define a distance between two observations is a priori unknown and must be estimated at each step using the available data. As a result, the matrices $B_{n}$ are no longer independent. Corollary 3 in Subsection 3.1 deals with this issue and thus broadens the scope of these processes. In Subsection 3.2, we define a type of processes that can be updated at each step with all previously observed data without the need for their storage. The conducted experiments show that this type of processes performs better than the former.

PCA is essential for data compression and feature extraction and has applications in various fields, such as data mining, engineering [23], face recognition [9], astronomy [7], text analytics [11, 17], etc. Batch PCA is unfeasible with massive datasets or data streams although online PCA algorithms which provide very fast updates can be used in this context [9]. Let $Z^{1}, \ldots, Z^{p}$ be the components of a random vector $Z$ and $C$ its covariance matrix. Define a metric $M$ in $\mathbb{R}^{p}$ and consider the following problem: for $l \in\{1, \ldots, r\}, r \leq p$, determine at step $l$ a linear combination of all the centered components of $Z, U_{l}=c_{l}^{\top}(Z-E[Z])$, named $l^{\text {th }}$ principal component, uncorrelated with $U_{1}, \ldots, U_{l-1}$ and of maximum variance under the constraint $c_{l}^{\top} M^{-1} c_{l}=1$. It is proven that $c_{l}$ is an eigenvector of the matrix $B=M C$ corresponding to its $l^{\text {th }}$ largest eigenvalue $\lambda_{l}$. For $l \in\{1, \ldots, r\}$, a $M$-normed direction vector $u_{l}$ of the $l^{\text {th }}$ principal axis is defined as $M^{-1} c_{l}$; the vectors $u_{l}$ are $M$-orthonormal and are eigenvectors of the matrix $C M$ corresponding respectively to the same eigenvalues $\lambda_{l}$. A particular case is normed PCA, where $M$ is the diagonal matrix of the inverses of variances of the $p$ components of $Z$; this is equivalent to using standardized data, i.e. observations of $M^{\frac{1}{2}}(Z-E[Z])$, and the identity metric, hence the same importance is given to each component of $Z$. However, in the case of streaming data, the expectation and the variance of the components of $Z$ are unknown. An application of this work is to recursively estimate the $c_{l}$ or the $u_{l}$ in this setting using a stochastic approximation process. Another example where the expectation of $Z$ and the metric used are unknown in the context of streaming data is generalized canonical correlation analysis (gCCA) [10, 18], which can be interpreted as a PCA where each pre-defined group of components of $Z$ has the same importance (see Section 5).

In order to reduce computing time and to avoid possible numerical explosions, we propose herein:

(a) to estimate the eigenvectors $a_{l}$ of the symmetric $(p, p)$ matrix $B=M^{\frac{1}{2}} C M^{\frac{1}{2}}$ (thus the Gram-Schmidt orthonormalization is made with respect to $Q=I$ ) and then, using an estimation of $M^{\frac{1}{2}}$ or $M^{-\frac{1}{2}}$, to deduce estimations of the eigenvectors $c_{l}$ of $M C$ or of the eigenvectors $u_{l}$ of $C M$, since $c_{l}=M^{\frac{1}{2}} a_{l}$ and $u_{l}=M^{-\frac{1}{2}} a_{l}$; note that if $M$ is the diagonal matrix of the inverses of variances of the $p$ components of $Z, B$ is the correlation matrix of $Z$;

(b) to replace $Z$ by $Z^{c}=Z-\xi$, $\xi$ being an estimation of $E[Z]$ computed in a preliminary phase with a small number of observations e.g. 1000, in order to reduce possible high values of certain components of $Z$ and to possibly avoid a numerical explosion; then, $B=M^{\frac{1}{2}}\left(E\left[Z^{c}\left(Z^{c}\right)^{\top}\right]-E\left[Z^{c}\right] E\left[Z^{c}\right]^{\top}\right) M^{\frac{1}{2}}$;

(c) to use, instead of a mini-batch of observations of $Z$ at step $n$, all observations up to this step without having to store them; thus information contained in previous data is used at each step; another algorithm with the same goal is History PCA [24].

Let $\left(Z_{11}, \ldots, Z_{1 m_{1}}, \ldots, Z_{n 1}, \ldots, Z_{n m_{n}}, \ldots\right)$ be an i.i.d. sample of $Z$. The variables $Z_{n j}, j \in\left\{1, \ldots, m_{n}\right\}$, are observed at time $n$. Let $\bar{Z}_{n-1}$ be the mean of the sample $\left(Z_{11}, \ldots, Z_{n-1, m_{n-1}}\right)$ of $Z$ and $M_{n-1}$ a $T_{n}$-measurable estimation of $M$ obtained from this sample. Let $Z_{n i}^{c}=Z_{n i}-\xi$ and $\bar{Z}_{n-1}^{c}=\bar{Z}_{n-1}-\xi$.

Given $\left(B_{n}\right)$, we recursively define the processes $\left(X_{n}^{i}\right), i \in\{1, \ldots, r\}$, by:

$$
Y_{n+1}^{i}=\left(I+a_{n} B_{n}\right) X_{n}^{i}, T_{n+1}^{i}=Y_{n+1}^{i}-\sum_{j<i}\left\langle Y_{n+1}^{i}, X_{n+1}^{j}\right\rangle X_{n+1}^{j}, X_{n+1}^{i}=\frac{T_{n+1}^{i}}{\left\|T_{n+1}^{i}\right\|} .
$$

As mentioned in (a), the Gram-Schmidt orthonormalization is made with respect to the metric $I$.

\subsection{Use of a data mini-batch at each step}

Let $B_{n}=M_{n-1}^{\frac{1}{2}}\left(\frac{1}{m_{n}} \sum_{j=1}^{m_{n}} Z_{n j}^{c}\left(Z_{n j}^{c}\right)^{\top}-\bar{Z}_{n-1}^{c}\left(\bar{Z}_{n-1}^{c}\right)^{\top}\right) M_{n-1}^{\frac{1}{2}}$. Then the conditional expectation

$$
E\left[B_{n} \mid T_{n}\right]=M_{n-1}^{\frac{1}{2}}\left(E\left[Z^{c}\left(Z^{c}\right)^{\top}\right]-\bar{Z}_{n-1}^{c}\left(\bar{Z}_{n-1}^{c}\right)^{\top}\right) M_{n-1}^{\frac{1}{2}}
$$

is different from $B$ but converges a.s. to $B$ under assumptions H6a and $\mathrm{H} 7 \mathrm{~b}$ given below: 
(H3') $a_{n}>0, \sum_{1}^{\infty} a_{n}=\infty, \sum_{1}^{\infty} \frac{a_{n}}{\sqrt{n}}<\infty, \sum_{1}^{\infty} a_{n}^{2}<\infty$;

(H6a) $\|Z\|$ is a.s. bounded;

(H6b) There is no affine or quadratic relationship between the components of $Z$;

(H7a) There exists a positive number $d$ such that $\sup _{n}\left\|M_{n}^{\frac{1}{2}}\right\|<d$ a.s.;

(H7b) $M_{n}^{\frac{1}{2}} \longrightarrow M^{\frac{1}{2}}$ a.s.;

(H7c) $\sum_{1}^{\infty} a_{n} E\left[\left\|M_{n-1}^{\frac{1}{2}}-M^{\frac{1}{2}}\right\|\right]<\infty$.

Corollary 3. Suppose assumptions H1b', H3', H5, H6a,b and H7a,b,c hold. Then, for $i \in\{1, \ldots, r\}, X_{n}^{i}$ converges a.s. to $V_{i}$ or $-V_{i}, \sum_{n=1}^{\infty} a_{n}\left|\lambda_{i}-\left(X_{n}^{i}\right)^{\prime} B X_{n}^{i}\right|$ and $\sum_{n=1}^{\infty} a_{n}\left(\lambda_{i}-\left(X_{n}^{i}\right)^{\prime} B_{n} X_{n}^{i}\right)$ converge a.s.

The proof is provided in Section 5.

\subsection{Use of all observations up to the current step}

Let $B_{n}=M_{n}^{\frac{1}{2}}\left(\frac{1}{\sum_{i=1}^{n} m_{i}} \sum_{i=1}^{n} \sum_{j=1}^{m_{i}} Z_{i j}^{c}\left(Z_{i j}^{c}\right)^{\top}-\bar{Z}_{n}^{c}\left(\bar{Z}_{n}^{c}\right)^{\top}\right) M_{n}^{\frac{1}{2}}$. Then the conditional expectation $E\left[B_{n} \mid T_{n}\right]$ is different from $B$, but $B_{n}$ converges a.s. to $B$ under assumptions $\mathrm{H} 7 \mathrm{~b}$ and $\mathrm{H} 6 \mathrm{c}$ below:

(H6c) $Z$ has $4^{\text {th }}$ order moments;

(H7d) $\sum_{1}^{\infty} a_{n}\left\|M_{n}^{\frac{1}{2}}-M^{\frac{1}{2}}\right\|<\infty$ a.s.

Corollary 4. Suppose assumptions $H 1 b^{\prime}, H 3^{\prime}, H 5^{\prime}, H 6 c, H 7 b, d$ hold. Then, for $i \in\{1, \ldots, r\}, X_{n}^{i}$ converges a.s. to $V_{i}$ or $-V_{i}, \sum_{1}^{\infty} a_{n}\left|\lambda_{i}-\left\langle X_{n}^{i}, B X_{n}^{i}\right\rangle\right|$ and $\sum_{1}^{\infty} a_{n}\left|\lambda_{i}-\left\langle X_{n}^{i}, B_{n} X_{n}^{i}\right\rangle\right|$ converge a.s.

The proof is provided in Section 5 .

Let $M$ be the diagonal matrix of the inverses of variances of $Z^{1}, \ldots, Z^{p}$. Let for $j \in\{1, \ldots, p\}, V_{n}^{j}$ be the empirical variance of the sample $\left(Z_{11}^{j}, \ldots, Z_{n, m_{n}}^{j}\right)$ of $Z^{j}$ and $M_{n}$ the diagonal matrix of order $p$ whose element $(j, j)$ is the inverse of $\frac{\mu_{n}}{\mu_{n}-1} V_{n}^{j}, \mu_{n}=\sum_{1}^{n} m_{i}$. Under H6c, H7b holds. Moreover, it is established in ([12], Lemma 5) that H7d holds under $\mathrm{H} 6 \mathrm{c}$ and $\mathrm{H} 3$ '.

\subsection{Widening to related methods}

The calculation of $M_{n-1}^{\frac{1}{2}}$ is straightforward when $M_{n-1}$ as $M$ are diagonal matrices. This is often the case in PCA and related methods such as normed PCA, Multiple Correspondence Analysis (MCA) [14] for categorical variables, Factor Analysis of Mixed Data (FAMD) [21] for continuous or categorical variables, and Multiple Factor Analysis (MFA) [21] for groups of continuous or categorical variables. However, in generalized Canonical Correlation Analysis (gCCA) [10], the metric $M$ is block diagonal (see Section 5). We can define $B_{n}$ in four different ways,

$$
B_{n}=M_{n-1}\left(\frac{1}{m_{n}} \sum_{j=1}^{m_{n}} Z_{n j}^{c}\left(Z_{n j}^{c}\right)^{\top}-\bar{Z}_{n-1}^{c}\left(\bar{Z}_{n-1}^{c}\right)^{\top}\right), B_{n}=M_{n}\left(\frac{1}{\sum_{i=1}^{n} m_{i}} \sum_{i=1}^{n} \sum_{j=1}^{m_{i}} Z_{i j}^{c}\left(Z_{i j}^{c}\right)^{\top}-\bar{Z}_{n}^{c}\left(\bar{Z}_{n}^{c}\right)^{\top}\right)
$$

and achieve the orthogonalization in processes $\left(X_{n}^{i}\right)$ with respect to $M_{n-1}^{-1}$ in the first case or $M_{n}^{-1}$ in the second case to estimate directly the eigenvectors $c_{i}$ of the $M^{-1}$ - symmetric matrix $B=M C$, or

$$
B_{n}=\left(\frac{1}{m_{n}} \sum_{j=1}^{m_{n}} Z_{n j}^{c}\left(Z_{n j}^{c}\right)^{\top}-\bar{Z}_{n-1}^{c}\left(\bar{Z}_{n-1}^{c}\right)^{\top}\right) M_{n-1}, B_{n}=\left(\frac{1}{\sum_{i=1}^{n} m_{i}} \sum_{i=1}^{n} \sum_{j=1}^{m_{i}} Z_{i j}^{c}\left(Z_{i j}^{c}\right)^{\top}-\bar{Z}_{n}^{c}\left(\bar{Z}_{n}^{c}\right)^{\top}\right) M_{n}
$$

and achieve the orthogonalization in processes $\left(X_{n}^{i}\right)$ with respect to $M_{n-1}$ in the third case or $M_{n}$ in the fourth case to estimate directly the eigenvectors $u_{i}$ of the $M$-symmetric matrix $B=C M, M_{n}^{\frac{1}{2}}$ being replaced by $M_{n}$ and $M^{\frac{1}{2}}$ by $M$ in assumptions H7a,b,c,d. This requires an extension of Theorem 1 and Corollary 2, the metric $Q\left(M^{-1}\right.$ or $\left.M\right)$ being replaced at step $n$ by an estimated $T_{n}$-measurable metric $Q_{n}$ such that $Q_{n}$ converges a.s. to $Q$ and $\sum_{n=1}^{\infty} a_{n}\left\|Q_{n}-Q\right\|<\infty$ a.s. This will be treated in a forthcoming article. 


\section{Conclusion}

In this article, we have provided almost sure convergence theorems of an Oja-type stochastic approximation process to eigenvectors of a $Q$-symmetric matrix $B$ corresponding to eigenvalues in decreasing order. These theorems apply to cases where there is a sequence $\left(B_{n}\right)$ such that $E\left[B_{n} \mid T_{n}\right]$ or $B_{n}$ converges a.s. to $B$. This extends previous results where random matrices $B_{n}$ are assumed to be i.i.d. and $E\left[B_{n} \mid T_{n}\right]=B$.

We subsequently applied these results to the online estimation of principal components in streaming PCA. By the streaming nature of the data, the expectation, the variance of the variables (or other characteristics if necessary) and the metric are generally unknown and need to be estimated online along with the principal components. Classical results of convergence do not apply to this case.

Moreover, we have defined a type of processes which are updated at each step by all of the previously observed data without necessitating their storage in memory. Classical algorithms only use a data mini-batch at each step.

In addition, these results can be applied to other factorial methods such as online gCCA.

Experiments have been conducted to compare processes of the second type (A: using all observations up to the current step) to processes of the first type (B: using a mini-batch of observations) on simulated or real datasets. Results regarding computing time are provided in the Appendix. It appears that processes A are generally faster than processes B. Such behavior is not surprising since, intuitively, a greater quantity of data at each step enables the algorithm to converge faster.

\section{Proofs}

Generalized Canonical Correlation Analysis. Suppose the set of components of a random vector $Z$ in $\mathbb{R}^{p}$ is partitioned in $q$ sets of real random variables $\left\{Z^{k 1}, \ldots, Z^{k m_{k}}\right\}, k \in\{1, \ldots, q\}$. Let $Z^{k}$ be the random vector in $\mathbb{R}^{m_{k}}$ whose components are $Z^{k j}, j \in\left\{1, \ldots, m_{k}\right\}$. Assume there is no affine relationship between the components of $Z$. Let $C^{k}$ and $C$ be the covariance matrices of $Z^{k}$ and $Z$, respectively. Consider the following problem: for $l \in\{1, \ldots, r\}, r \leq p$, determine at step $l$ a linear combination of all the centered components of $Z, U_{l}=\theta_{l}^{\top}(Z-E[Z])$, named $l^{\text {th }}$ general component, of variance 1 and uncorrelated with $U_{1}, \ldots, U_{l-1}$, and, for $k \in\{1, \ldots, q\}$, a linear combination of variance 1 of the centered components of $Z^{k}, V_{l}^{k}=\left(\eta_{l}^{k}\right)^{\top}\left(Z^{k}-E\left[Z^{k}\right]\right)$, named $l^{\text {th }}$ canonical component of the $k^{\text {th }}$ set of variables, which maximize $\sum_{k=1}^{q} \rho^{2}\left(U_{l}, V_{l}^{k}\right), \rho$ denoting the linear correlation coefficient. Let $M$ be the unknown block diagonal matrix of order $p$ whose $k^{\text {th }}$ diagonal block is $\left(C^{k}\right)^{-1}$. Let $\theta_{l}=\left(\left(\theta_{l}^{1}\right)^{\top}, \ldots,\left(\theta_{l}^{q}\right)^{\top}\right)^{\top}, \theta_{l}^{k} \in \mathbb{R}^{m_{k}}, k \in\{1, \ldots, q\}$. It is proven that $\theta_{l}$ is a $C$-normed eigenvector of the $M^{-1}$-symmetric matrix $B=M C$ corresponding to its $l^{\text {th }}$ largest eigenvalue and that for $k \in\{1, \ldots, q\}$, there exists $\alpha_{l}^{k} \in \mathbb{R}$ such that $\eta_{l}^{k}=\alpha_{l}^{k} \theta_{l}^{k}$. Note that $\theta_{l}$ is collinear with the $l^{\text {th }}$ principal component of PCA of $Z$ with the metric $M$. The objective is then to perform online PCA of $Z$ using at step $n$ a consistent estimator $M_{n}$ of $M$.

Proof of Theorem 1, first part. Its plan follows that of ([13], Section 9.4.2, p. 343) in the case of PCA with $E\left[B_{n} \mid T_{n}\right]=$ $B$ a.s. Let $X_{n}^{j}=\left\langle X_{n}, V_{j}\right\rangle$. After establishing that there exists $K_{2}>0$ and $\beta_{n}$ such that a.s.

$$
X_{n+1}=\left(I+a_{n}\left(B_{n}-W_{n} I\right)+a_{n} \beta_{n}\right) X_{n},\left\|\beta_{n}\right\| \leq K_{2} a_{n},
$$

we prove that $U_{n}$ converges a.s., then that if the limit of $U_{n}$ is different from an eigenvalue $\lambda_{j}, X_{n}^{j}$ converges a.s. to 0 . Since $\left\|X_{n}\right\|=1$, this cannot be true for every $j$, therefore the limit of $U_{n}$ is one of the eigenvalues of $B, \lambda_{i}$, and $X_{n}$ converges to $V_{i}$ or $-V_{i}$ on $E_{i}=\left\{U_{n} \longrightarrow \lambda_{i}\right\}$. We then prove that $\sum_{n=1}^{\infty} a_{n}\left(\lambda_{i}-U_{n}\right)$ and $\sum_{n=1}^{\infty} a_{n}\left(\lambda_{i}-W_{n}\right)$ converge on $E_{i}$. Applying a lemma of Duflo ([13], p. 342) to the sequence $\left(X_{n}^{1}\right)$ on $E_{i}$, we prove that, if $\lim \inf E\left[\left\langle B_{n} X_{n}, V_{1}\right\rangle^{2}\right.$ $\left.\mid T_{n}\right]>0, P\left(E_{i}\right)=0$ for $i>1$. Therefore, $X_{n}$ converges a.s. to $V_{1}$.

Let us state two lemmas of Duflo ([13], p. 16, 342) used in this proof.

Lemma 5. Let $\left(M_{n}\right)$ be a square-integrable martingale adapted to a filtration $\left(T_{n}\right)$ and $\left(\langle M\rangle_{n}\right)$ its increasing process defined recursively by:

$$
\langle M\rangle_{1}=M_{1}^{2},\langle M\rangle_{n+1}=\langle M\rangle_{n}+E\left[\left(M_{n+1}-M_{n}\right)^{2} \mid T_{n}\right]=\langle M\rangle_{n}+E\left[M_{n+1}^{2} \mid T_{n}\right]-M_{n}^{2} .
$$

Let $\langle M\rangle_{\infty}=\lim \langle M\rangle_{n}$. If $E\left[\langle M\rangle_{\infty}\right]<\infty$, then $\left(M_{n}\right)$ converges a.s. and in mean square to a finite random variable. 
In the following lemma, Duflo provides a tool which can be used to prove that given a sufficiently exciting noise $\left(\epsilon_{n}\right)$, the traps of recursive algorithms of the type defined in assumption (i) below can be avoided.

Lemma 6. Let $\left(\gamma_{n}\right)$ be a sequence of positive numbers such that $\sum_{n=1}^{\infty} \gamma_{n}^{2}<\infty$. Let $\left(Z_{n}\right)$ and $\left(\delta_{n}\right)$ be two sequences of random variables adapted to a filtration $\left(T_{n}\right)$, and $\left(\epsilon_{n}\right)$ a noise adapted to $\left(T_{n}\right)$.

Assume on a set $\Gamma$ :

(i) for every integer $n, Z_{n+1}=Z_{n}\left(1+\delta_{n}\right)+\gamma_{n} \epsilon_{n+1}$;

(ii) $\left(Z_{n}\right)$ is bounded;

(iii) $\sum_{n=1}^{\infty} \delta_{n}^{2}<\infty, \delta_{n} \geq 0$ for $n$ sufficiently large, and there exists a sequence of positive numbers $\left(b_{n}\right)$ such that $\sum_{n=1}^{\infty} b_{n}=\infty$ and $\sum_{n=1}^{\infty}\left(b_{n}-\delta_{n}\right)$ converges;

(iv) for an $a>2, E\left[\left|\epsilon_{n+1}\right|^{a} \mid T_{n}\right]=O(1)$ and $\lim \inf E\left[\epsilon_{n+1}^{2} \mid T_{n}\right]>0$ a.s.

Then, $P(\Gamma)=0$.

Note that, since $\sup _{n}\left\|B_{n}\right\|<b$ a.s. (H2a) and $a_{n}$ converges to $0(\mathrm{H} 3), I+a_{n} B_{n}$ is invertible from a certain rank $N$, and if $X_{N}$ is different from $0,\left\|\left(I+a_{n} B_{N}\right) X_{N}\right\| \neq 0$, thus $X_{n}$ is defined for all $n \geqslant N$ and $\left\|X_{n}\right\|=1$. We have $\left\|U_{n}\right\| \leq\|B\|,\left\|W_{n}\right\| \leq\left\|B_{n}\right\| \leq b$ a.s. Let $K_{i}, i=1,2,3,4,5$ be adequately chosen real numbers. Under H2a, since $\left\|\left(I+a_{n} B_{n}\right) X_{n}\right\|^{2}=1+2 a_{n} W_{n}+a_{n}^{2}\left\|B_{n} X_{n}\right\|^{2}$, we have almost surely:

$$
\begin{aligned}
\frac{1}{\left\|\left(I+a_{n} B_{n}\right) X_{n}\right\|} & =1-a_{n} W_{n}-\frac{1}{2} a_{n}^{2}\left\|B_{n} X_{n}\right\|^{2}+\alpha_{n}, \quad\left|\alpha_{n}\right| \leq K_{1} a_{n}^{2}, \\
X_{n+1} & =\left(I+a_{n} B_{n}\right)\left(1-a_{n} W_{n}-\frac{1}{2} a_{n}^{2}\left\|B_{n} X_{n}\right\|^{2}+\alpha_{n}\right) X_{n}=\left(I+a_{n}\left(B_{n}-W_{n} I\right)+a_{n} \beta_{n}\right) X_{n}, \\
\beta_{n} & =-a_{n} W_{n} B_{n}-\frac{1}{2} a_{n}\left\|B_{n} X_{n}\right\|^{2} I-\frac{1}{2} a_{n}^{2} B_{n}\left\|B_{n} X_{n}\right\|^{2}+a_{n}^{-1} \alpha_{n} I+\alpha_{n} B_{n},\left\|\beta_{n}\right\| \leq K_{2} a_{n}, \\
X_{n+1} & =\left(I+a_{n}\left(B-U_{n} I\right)+a_{n} \Gamma_{n}\right) X_{n}, \Gamma_{n}=\left(B_{n}-B\right)-\left\langle X_{n},\left(B_{n}-B\right) X_{n}\right\rangle I+\beta_{n},\left\|\Gamma_{n}\right\| \leq K_{3} .
\end{aligned}
$$

\section{Step 1: convergence of $\mathbf{U}_{n}$.}

$$
\begin{aligned}
U_{n+1} & =\left\langle\left(I+a_{n}\left(B-U_{n} I\right)+a_{n} \Gamma_{n}\right) X_{n}, B\left(I+a_{n}\left(B-U_{n} I\right)+a_{n} \Gamma_{n}\right) X_{n}\right\rangle \\
& =U_{n}+2 a_{n}\left\langle\left(B-U_{n} I\right) X_{n}, B X_{n}\right\rangle+2 a_{n}\left\langle\Gamma_{n} X_{n}, B X_{n}\right\rangle+a_{n}^{2} \eta_{n} \\
\eta_{n} & =\left\langle\left(B-U_{n} I\right) X_{n}, B\left(B-U_{n} I\right) X_{n}\right\rangle+2\left\langle\Gamma_{n} X_{n}, B\left(B-U_{n} I\right) X_{n}\right\rangle+\left\langle\Gamma_{n} X_{n}, B \Gamma_{n} X_{n}\right\rangle,\left|\eta_{n}\right| \leq K_{4} \text { a.s. }
\end{aligned}
$$

Let $\mu_{n}=2 a_{n}\left\langle E\left[\Gamma_{n} \mid T_{n}\right] X_{n}, B X_{n}\right\rangle+a_{n}^{2} E\left[\eta_{n} \mid T_{n}\right]$. Since $\left\|X_{n}\right\|=1$ :

$$
\left\langle\left(B-U_{n} I\right) X_{n}, B X_{n}\right\rangle=\left\|B X_{n}\right\|^{2}-U_{n}^{2}=\left\|B X_{n}-U_{n} X_{n}\right\|^{2} \geqslant 0 .
$$

Then, $E\left[U_{n+1} \mid T_{n}\right] \geq U_{n}+\mu_{n}$ and $E\left[U_{n+1}-\sum_{i=1}^{n} \mu_{i} \mid T_{n}\right] \geq U_{n}-\sum_{i=1}^{n-1} \mu_{i}$ a.s., thus $U_{n}-\sum_{i=1}^{n-1} \mu_{i}$ is a submartingale. Since $\left\|\beta_{n}\right\| \leq K_{2} a_{n}$ and $\left|\eta_{n}\right| \leq K_{4}$ :

$$
\begin{aligned}
\left\|E\left[\Gamma_{n} \mid T_{n}\right]\right\| & \leq 2\left\|E\left[B_{n} \mid T_{n}\right]-B\right\|+\left\|E\left[\beta_{n} \mid T_{n}\right]\right\| ; \\
\left|\mu_{n}\right| & \leq 4 a_{n}\left\|E\left[B_{n} \mid T_{n}\right]-B\right\|+2 a_{n}\left\|E\left[\beta_{n} \mid T_{n}\right]\right\|+K_{4} a_{n}^{2} \leq 4 a_{n}\left\|E\left[B_{n} \mid T_{n}\right]-B\right\|+K_{5} a_{n}^{2} \text { a.s. }
\end{aligned}
$$

By H2b $\left(\sum_{n=1}^{\infty} a_{n} E\left[\left\|E\left[B_{n} \mid T_{n}\right]-B\right\|\right]<\infty\right)$ and H3, we have: $\sup _{n} E\left[\left|\sum_{i=1}^{n-1} \mu_{i}\right|\right]<\infty$. By Doob's lemma, the submartingale $U_{n}-\sum_{i=1}^{n-1} \mu_{i}$ converges a.s. to an integrable random variable. Since $\sum_{i=1}^{n-1} \mu_{i}$ converges, $U_{n}$ converges a.s.

Step 2: convergence of $\mathbf{X}_{n}^{j}=\left\langle\mathbf{X}_{n}, \mathbf{V}_{j}\right\rangle$. Let $\Gamma_{n}^{j}=\left\langle\Gamma_{n} X_{n}, V_{j}\right\rangle$. Since $B$ is $Q$-symmetric, $\left\langle B X_{n}, V_{j}\right\rangle=\lambda_{j} X_{n}^{j}$.

$$
\begin{aligned}
X_{n+1}^{j} & =\left\langle\left(I+a_{n}\left(B-U_{n} I\right)+a_{n} \Gamma_{n}\right) X_{n}, V_{j}\right\rangle=X_{n}^{j}+a_{n}\left(\left(\lambda_{j}-U_{n}\right) X_{n}^{j}+\Gamma_{n}^{j}\right) ; \\
\left(X_{n+1}^{j}\right)^{2} & =\left(X_{n}^{j}\right)^{2}+a_{n}^{2}\left(\left(\lambda_{j}-U_{n}\right) X_{n}^{j}+\Gamma_{n}^{j}\right)^{2}+2 a_{n}\left(\lambda_{j}-U_{n}\right)\left(X_{n}^{j}\right)^{2}+2 a_{n} X_{n}^{j} \Gamma_{n}^{j} \\
& =\left(X_{1}^{j}\right)^{2}+\sum_{l=1}^{n} a_{l}^{2}\left(\left(\lambda_{j}-U_{l}\right) X_{l}^{j}+\Gamma_{l}^{j}\right)^{2}+2 \sum_{l=1}^{n} a_{l} X_{l}^{j} \Gamma_{l}^{j}+2 \sum_{l=1}^{n} a_{l}\left(\lambda_{j}-U_{l}\right)\left(X_{l}^{j}\right)^{2} .
\end{aligned}
$$


Prove the convergence of the three last terms of this decomposition.

(i) Since $\left\|U_{l}\right\| \leq\|B\|$ and $\left\|\Gamma_{l}\right\| \leq K_{3}$ a.s., $\sum_{l=1}^{\infty} a_{l}^{2}\left(\left(\lambda_{j}-U_{l}\right) X_{l}^{j}+\Gamma_{l}^{j}\right)^{2}$ converges a.s. by $\mathrm{H} 3$.

(ii) Let $M_{n}^{j}=\sum_{l=1}^{n-1} a_{l} X_{l}^{j}\left(\Gamma_{l}^{j}-E\left[\Gamma_{l}^{j} \mid T_{l}\right]\right)$.

$\sum_{l=1}^{n} a_{l} X_{l}^{j} \Gamma_{l}^{j}=\sum_{l=1}^{n} a_{l} X_{l}^{j}\left(\Gamma_{l}^{j}-E\left[\Gamma_{l}^{j} \mid T_{l}\right]\right)+\sum_{l=1}^{n} a_{l} X_{l}^{j} E\left[\Gamma_{l}^{j} \mid T_{l}\right]=\sum_{l=1}^{n} a_{l} X_{l}^{j} E\left[\Gamma_{l}^{j} \mid T_{l}\right]+M_{n+1}^{j}$.

Firstly: $\sum_{l=1}^{n} a_{l}\left|X_{l}^{j} E\left[\Gamma_{l}^{j} \mid T_{l}\right]\right| \leq \sum_{l=1}^{n} a_{l}\left\|E\left[\Gamma_{l} \mid T_{l}\right]\right\| \leq \sum_{l=1}^{n} a_{l}\left(2\left\|E\left[B_{l} \mid T_{l}\right]-B\right\|+\left\|E\left[\beta_{l} \mid T_{l}\right]\right\|\right)$.

By $\mathrm{H} 2 \mathrm{~b}\left(\sum_{n=1}^{\infty} a_{n} E\left[\left\|E\left[B_{n} \mid T_{n}\right]-B\right\|\right]<\infty\right)$ and $\mathrm{H} 3$, since $\left\|\beta_{n}\right\| \leq K_{2} a_{n}$ a.s., $\sum_{l=1}^{\infty} a_{l} X_{l}^{j} E\left[\Gamma_{l}^{j} \mid T_{l}\right]$ converges a.s.

Secondly: $\left(M_{n}^{j}\right)$ is a square-integrable martingale adapted to the filtration $\left(T_{n}\right)$; let $\left(\left\langle M^{j}\right\rangle_{n}\right)$ be its increasing process. $\left\langle M^{j}\right\rangle_{n+1}-\left\langle M^{j}\right\rangle_{n}=E\left[\left(M_{n+1}^{j}-M_{n}^{j}\right)^{2} \mid T_{n}\right]=a_{n}^{2} E\left[\left(X_{n}^{j}\right)^{2}\left(\Gamma_{n}^{j}-E\left[\Gamma_{n}^{j} \mid T_{n}\right]\right)^{2} \mid T_{n}\right] \leq K_{3} a_{n}^{2}$.

Then, applying Lemma 5, since $E\left[\langle M\rangle_{\infty}\right]<\infty$ by H3, $\left(M_{n}^{j}\right)$ converges a.s. to a finite random variable. Thus, $\sum_{l=1}^{\infty} a_{l} X_{l}^{j} \Gamma_{l}^{j}$ converges a.s.

(iii) Let $\omega$ be fixed belonging to the convergence set of $U_{n}$. The writing of $\omega$ will be omitted in the following. Let $L$ be the limit of $U_{n}$. If $L \neq \lambda_{j}$, the sign of $\lambda_{j}-U_{n}$ is constant from a certain rank $N$ depending on $\omega$. Using the decomposition of $\left(X_{n+1}^{j}\right)^{2}$, there exists $A>0$ such that:

$$
\sum_{l=N}^{n} a_{l}\left|\lambda_{j}-U_{l}\right|\left(X_{l}^{j}\right)^{2}=\left|\sum_{l=N}^{n} a_{l}\left(\lambda_{j}-U_{l}\right)\left(X_{l}^{j}\right)^{2}\right|=\left|\left(X_{n+1}^{j}\right)^{2}-\left(X_{N}^{j}\right)^{2}-\sum_{l=N}^{n} a_{l}^{2}\left(\left(\lambda_{j}-U_{l}\right) X_{l}^{j}+\Gamma_{l}^{j}\right)^{2}-2 \sum_{l=N}^{n} a_{l} X_{l}^{j} \Gamma_{l}^{j}\right|<A
$$

Then for $L \neq \lambda_{j}, \sum_{l=N}^{\infty} a_{l}\left|\lambda_{j}-U_{l}\right|\left(X_{l}^{j}\right)^{2}$ and $\sum_{l=N}^{\infty} a_{l}\left(\lambda_{j}-U_{l}\right)\left(X_{l}^{j}\right)^{2}$ converge.

It follows from (i), (ii) and (iii) that for $L \neq \lambda_{j},\left(X_{n}^{j}\right)^{2}$ converges a.s. Since by (iii), $\sum_{l=1}^{\infty} a_{l}\left(X_{l}^{j}\right)^{2}<\infty, X_{n}^{j}$ converges a.s. to 0 . Since $\left\|X_{n}\right\|=1$, this cannot be true for every $j$. Thus the limit of $U_{n}$ is one of the eigenvalues of B, $\lambda_{i}$.

Step 3: convergence of $\mathbf{X}_{n}$. On $E_{i}=\left\{U_{n} \longrightarrow \lambda_{i}\right\}$, for $j \neq i, X_{n}^{j}$ converges to 0 , therefore $\left(X_{n}^{i}\right)^{2}$ converges to 1 and since

$$
X_{n+1}-X_{n}=a_{n}\left(\left(B-U_{n} I\right)+\Gamma_{n}\right) X_{n},
$$

$X_{n+1}-X_{n}$ converges to 0 and the limit of $X_{n}$ is $V_{i}$ or $-V_{i}$ on $E_{i}$ (first assertion of Theorem 1 ).

Using the decomposition of $\left(X_{n}^{i}\right)^{2}$ in Step 2, the convergence of $\left(X_{n}^{i}\right)^{2}$ and of (i) and (ii) yields that $\sum_{n=1}^{\infty} a_{n}\left(\lambda_{i}-U_{n}\right)$ converges a.s. on $E_{i}$ (first assertion of Theorem 1). Consider now the decomposition:

$$
\sum_{n=1}^{\infty} a_{n}\left(\lambda_{i}-W_{n}\right)=\sum_{n=1}^{\infty} a_{n}\left(\lambda_{i}-U_{n}\right)+\sum_{n=1}^{\infty} a_{n}\left\langle X_{n},\left(B_{n}-E\left[B_{n} \mid T_{n}\right]\right) X_{n}\right\rangle+\sum_{n=1}^{\infty} a_{n}\left\langle X_{n},\left(E\left[B_{n} \mid T_{n}\right]-B\right) X_{n}\right\rangle .
$$

Firstly, since $\sum_{n=1}^{\infty} a_{n} E\left[\left\|E\left[B_{n} \mid T_{n}\right]-B\right\|\right]<\infty(\mathrm{H} 2 \mathrm{~b}), \sum_{n=1}^{\infty} a_{n}\left\langle X_{n},\left(E\left[B_{n} \mid T_{n}\right]-B\right) X_{n}\right\rangle$ converges a.s.

Secondly, let $M_{n}=\sum_{l=1}^{n-1} a_{l}\left\langle X_{l},\left(B_{l}-E\left[B_{l} \mid T_{l}\right]\right) X_{l}\right\rangle ;\left(M_{n}\right)$ is a square-integrable martingale adapted to the filtration $\left(T_{n}\right)$. Its increasing process $\left(\langle M\rangle_{n}\right)$ converges: indeed, since $\sup _{n}\left\|B_{n}\right\|<b$ a.s.,

$$
\begin{aligned}
\langle M\rangle_{n+1}-\langle M\rangle_{n} & =E\left[\left(M_{n+1}-M_{n}\right)^{2} \mid T_{n}\right]=a_{n}^{2} E\left[\left\langle X_{n},\left(B_{n}-E\left[B_{n} \mid T_{n}\right]\right) X_{n}\right\rangle^{2} \mid T_{n}\right] \\
& \leq a_{n}^{2} E\left[\left\|B_{n}-E\left[B_{n} \mid T_{n}\right]\right\|^{2} \mid T_{n}\right] \leq a_{n}^{2} E\left[\left\|B_{n}\right\|^{2} \mid T_{n}\right] \leq b^{2} a_{n}^{2} .
\end{aligned}
$$

Thus by $\mathrm{H} 3$ and Lemma $5,\left(M_{n}\right)$ converges a.s. to a finite random variable. Therefore, $\sum_{n=1}^{\infty} a_{n}\left(\lambda_{i}-W_{n}\right)$ converges a.s. on $E_{i}$ (first assertion of Theorem 1).

Step 4: convergence of $\mathbf{X}_{n}$ to $\pm \mathbf{V}_{1}$. Suppose $i>1$. Recall that, with $\Gamma_{n}=\left(B_{n}-B\right)-\left\langle X_{n},\left(B_{n}-B\right) X_{n}\right\rangle I+\beta_{n}$ :

$$
X_{n+1}^{1}=\left(1+a_{n}\left(\lambda_{1}-U_{n}\right)\right) X_{n}^{1}+a_{n}\left\langle\Gamma_{n} X_{n}, V_{1}\right\rangle=\left(1+a_{n}\left(\left(\lambda_{1}-\lambda_{i}\right)+\left(\lambda_{i}-U_{n}\right)\right)\right) X_{n}^{1}+a_{n}\left\langle\Gamma_{n} X_{n}, V_{1}\right\rangle .
$$

In the following, apply Lemma 6 to the sequence $\left(X_{n}^{1}\right)$ on $E_{i}=\left\{X_{n} \longrightarrow V_{i}\right\}, i>1$, with $\gamma_{n}=a_{n}, \delta_{n}=a_{n}\left(\lambda_{1}-U_{n}\right)$, $b_{n}=a_{n}\left(\lambda_{1}-\lambda_{i}\right)>0, \epsilon_{n+1}=\left\langle\Gamma_{n} X_{n}, V_{1}\right\rangle$. Verify the assumptions (ii), (iii) and (iv) of Lemma 6:

(ii) $X_{n}^{1}$ is bounded;

(iii) $\sum_{n=1}^{\infty} a_{n}^{2}<\infty, \sum_{n=1}^{\infty} a_{n}^{2}\left(\lambda_{1}-U_{n}\right)^{2}<\infty, \sum_{n=1}^{\infty} a_{n}\left(\lambda_{1}-\lambda_{i}\right)=\infty, \sum_{n=1}^{\infty} a_{n}\left(\lambda_{i}-U_{n}\right)$ converges a.s. on $E_{i}$;

(iv) $E\left[\left\langle\Gamma_{n} X_{n}, V_{1}\right\rangle^{2} \mid T_{n}\right] \geqslant \frac{1}{2} E\left[\left\langle B_{n} X_{n}, V_{1}\right\rangle^{2} \mid T_{n}\right]-E\left[\left\langle\left(\Gamma_{n}-B_{n}\right) X_{n}, V_{1}\right\rangle^{2} \mid T_{n}\right]$ a.s.

$$
\begin{aligned}
\left\langle\left(\Gamma_{n}-B_{n}\right) X_{n}, V_{1}\right\rangle & =-\left\langle B X_{n}, V_{1}\right\rangle-\left\langle\left(B_{n}-B\right) X_{n}, X_{n}\right\rangle\left\langle X_{n}, V_{1}\right\rangle+\left\langle\beta_{n} X_{n}, V_{1}\right\rangle \\
& =-X_{n}^{1}\left(\lambda_{1}+\left\langle\left(B_{n}-B\right) X_{n}, X_{n}\right\rangle\right)+\left\langle\beta_{n} X_{n}, V_{1}\right\rangle .
\end{aligned}
$$


Since $\left\|\Gamma_{n}\right\| \leq K_{3}$ and $\left\|\beta_{n}\right\| \leq K_{2} a_{n}$ a.s., there exists a positive number $c$ such that a.s. on $E_{i}$ :

$E\left[\left\langle\left(\Gamma_{n}-B_{n}\right) X_{n}, V_{1}\right\rangle^{2} \mid T_{n}\right] \leq c\left(X_{n}^{1}\right)^{2}+2 E\left[\left\|\beta_{n}\right\|^{2} \mid T_{n}\right] \leq c\left(X_{n}^{1}\right)^{2}+2 K_{2}^{2} a_{n}^{2} \underset{n \rightarrow+\infty}{\longrightarrow} 0$ since $X_{n}^{1} \underset{n \rightarrow+\infty}{\longrightarrow} 0$ on $E_{i}$.

Then, if liminf $E\left[\left\langle B_{n} X_{n}, V_{1}\right\rangle^{2} \mid T_{n}\right]>0, \liminf E\left[\left\langle\Gamma_{n} X_{n}, V_{1}\right\rangle^{2} \mid T_{n}\right]>0$. By Lemma $6, P\left(E_{i}\right)=0, i>1$.

Thus $P\left(E_{1}\right)=1$ (second assertion of Theorem 1 ).

Proof of Theorem 1, second part. Recursively define the processes $\left(\widetilde{X}_{n}\right)$ and $\left(\widetilde{U}_{n}\right)$ such that

$$
\begin{aligned}
& \widetilde{X}_{n+1}=\left(I+a_{n} B_{n}\right) \widetilde{X}_{n}, \widetilde{X}_{1}=X_{1}, \\
& \widetilde{U}_{n+1}=\frac{\widetilde{X}_{n+1}}{\prod_{i=1}^{n}\left(1+\lambda_{1} a_{i}\right)}=\frac{I+a_{n} B_{n}}{1+\lambda_{1} a_{n}} \widetilde{U}_{n}=\widetilde{U}_{n}+\frac{a_{n}}{1+\lambda_{1} a_{n}}\left(B_{n} \widetilde{U}_{n}-\lambda_{1} \widetilde{U}_{n}\right), \widetilde{U}_{1}=X_{1} .
\end{aligned}
$$

Note that $\frac{\widetilde{U}_{n}}{\left\|\widetilde{U}_{n}\right\|}=\frac{\widetilde{X}_{n}}{\left\|\widetilde{X}_{n}\right\|}=X_{n}$.

We prove in the first step that $\left\|\widetilde{U}_{n}\right\|$ and $\sum_{n=1}^{\infty} a_{n}\left\|\widetilde{U}_{n}\right\|^{2}\left(\lambda_{1}-\left\langle B X_{n}, X_{n}\right\rangle\right)$ converge a.s., in the second step that $\widetilde{U}_{n}^{j}=$ $\left\langle\widetilde{U}_{n}, V_{j}\right\rangle$ converges a.s. to $\widetilde{U}^{j}$ and that $\widetilde{U}^{j}=0$ for $j>1$, in the third step that $\widetilde{U}^{1} \neq 0$. The conclusion is then immediate.

Lemma 7. Suppose $\left(z_{n}, n \geqslant 1\right),\left(\alpha_{n}, n \geqslant 1\right),\left(\beta_{n}, n \geqslant 1\right)$ and $\left(\gamma_{n}, n \geqslant 1\right)$ are four sequences of non-negative numbers such that for all $n \geqslant 1, z_{n+1} \leq z_{n}\left(1+\alpha_{n}\right)+\beta_{n}-\gamma_{n}, \sum_{n=1}^{\infty} \alpha_{n}<\infty, \sum_{n=1}^{\infty} \beta_{n}<\infty$. Then the sequence $\left(z_{n}\right)$ converges and $\sum_{n=1}^{\infty} \gamma_{n}<\infty$.

This is a deterministic form of the Robbins-Siegmund lemma [22]. Its direct proof is based on the convergence of the decreasing sequence $\left(u_{n}\right)$ :

$$
u_{n}=\frac{z_{n}}{\prod_{l=1}^{n-1}\left(1+\alpha_{l}\right)}-\sum_{k=1}^{n-1} \frac{\beta_{k}-\gamma_{k}}{\prod_{l=1}^{k}\left(1+\alpha_{l}\right)} \geqslant-\sum_{k=1}^{\infty} \beta_{k} .
$$

Let $\omega$ be fixed, belonging to $C_{1}=\left\{\sum_{n=1}^{\infty} a_{n}\left\|B_{n}-B\right\|<\infty\right\}$. The writing of $\omega$ will be omitted in the following.

Step 1 Since $a_{n} \longrightarrow 0$ (H3), $1+\lambda_{1} a_{n}>0$ from a certain rank $N$. Suppose $N=1$ without loss of generality.

$$
\begin{aligned}
\left\|\widetilde{U}_{n+1}\right\|^{2} & =\left\|\widetilde{U}_{n}\right\|^{2}+2 \frac{a_{n}}{1+\lambda_{1} a_{n}}\left\langle\widetilde{U}_{n},\left(B_{n}-\lambda_{1} I\right) \widetilde{U}_{n}\right\rangle+\frac{a_{n}^{2}}{\left(1+\lambda_{1} a_{n}\right)^{2}}\left\|\left(B_{n}-\lambda_{1} I\right) \widetilde{U}_{n}\right\|^{2} \\
& =\left\|\widetilde{U}_{n}\right\|^{2}+2 \frac{a_{n}}{1+\lambda_{1} a_{n}}\left\langle\widetilde{U}_{n},\left(B_{n}-B\right) \widetilde{U}_{n}\right\rangle+\frac{a_{n}^{2}}{\left(1+\lambda_{1} a_{n}\right)^{2}}\left\|\left(B_{n}-\lambda_{1} I\right) \widetilde{U}_{n}\right\|^{2}-2 \frac{a_{n}}{1+\lambda_{1} a_{n}}\left\langle\widetilde{U}_{n},\left(\lambda_{1} I-B\right) \widetilde{U}_{n}\right\rangle .
\end{aligned}
$$

$\lambda_{1} I-B$ is a non-negative $Q$-symmetric matrix, with eigenvalues $0, \lambda_{1}-\lambda_{2}, \ldots, \lambda_{1}-\lambda_{p}$.

$$
\begin{aligned}
\left\|B_{n}-\lambda_{1} I\right\|^{2} \leq & 2\left\|B_{n}-B\right\|^{2}+2\left\|\lambda_{1} I-B\right\|^{2} . \\
\left\|\widetilde{U}_{n+1}\right\|^{2} \leq & \left\|\widetilde{U}_{n}\right\|^{2}\left(1+2 \frac{a_{n}}{1+\lambda_{1} a_{n}}\left\|B_{n}-B\right\|+2 \frac{a_{n}^{2}}{\left(1+\lambda_{1} a_{n}\right)^{2}}\left\|B_{n}-B\right\|^{2}+2 \frac{a_{n}^{2}}{\left(1+\lambda_{1} a_{n}\right)^{2}}\left(\lambda_{1}-\lambda_{p}\right)^{2}\right) \\
& -2 \frac{a_{n}}{1+\lambda_{1} a_{n}}\left\langle\widetilde{U}_{n},\left(\lambda_{1} I-B\right) \widetilde{U}_{n}\right\rangle .
\end{aligned}
$$

By assumptions $\mathrm{H} 2 \mathrm{~b}$ ' and $\mathrm{H} 3$, applying Lemma 7 yields:

$$
\left\|\widetilde{U}_{n}\right\|^{2} \underset{n \rightarrow+\infty}{\longrightarrow} \widetilde{U}, \quad \sum_{n=1}^{\infty} a_{n}\left\langle\widetilde{U}_{n},\left(\lambda_{1} I-B\right) \widetilde{U}_{n}\right\rangle=\sum_{n=1}^{\infty} a_{n}\left\|\widetilde{U}_{n}\right\|^{2}\left(\lambda_{1}-\frac{\left\langle\widetilde{U}_{n}, B \widetilde{U}_{n}\right\rangle}{\left\|\widetilde{U}_{n}\right\|^{2}}\right)<\infty .
$$

Since $\sum_{n=1}^{\infty} a_{n}=\infty$, either $\left\|\widetilde{U}_{n}\right\|_{n \rightarrow+\infty}^{\longrightarrow} 0$ or $\sum_{n=1}^{\infty} a_{n}\left(\lambda_{1}-\left\langle X_{n}, B X_{n}\right\rangle\right)<\infty$.

Step 2: Let $\widetilde{U}_{n}^{j}=\left\langle\widetilde{U}_{n}, V_{j}\right\rangle$.

$$
\begin{aligned}
\widetilde{U}_{n+1}^{j} & =\left\langle V_{j}, \frac{I+a_{n} B_{n}}{1+\lambda_{1} a_{n}} \widetilde{U}_{n}\right\rangle=\left\langle V_{j}, \frac{1}{1+\lambda_{1} a_{n}}\left(I+a_{n} B+a_{n}\left(B_{n}-B\right)\right) \widetilde{U}_{n}\right\rangle \\
& =\frac{1+\lambda_{j} a_{n}}{1+\lambda_{1} a_{n}} \widetilde{U}_{n}^{j}+\frac{a_{n}}{1+\lambda_{1} a_{n}}\left\langle V_{j},\left(B_{n}-B\right) \widetilde{U}_{n}\right\rangle .
\end{aligned}
$$


(a) For $j>1$, since $a_{n} \underset{n \rightarrow+\infty}{\longrightarrow} 0$ and $\lambda_{1}-\lambda_{j}>0$, there exists $\alpha_{n}=O\left(a_{n}\right)>0$ such that for $n$ sufficiently large:

$$
\left|\widetilde{U}_{n+1}^{j}\right| \leq \frac{1+\lambda_{j} a_{n}}{1+\lambda_{1} a_{n}}\left|\widetilde{U}_{n}^{j}\right|+\frac{a_{n}}{1+\lambda_{1} a_{n}}\left\|B_{n}-B\right\|\left\|\widetilde{U}_{n}\right\| \leq\left(1-\alpha_{n}\right)\left|\widetilde{U}_{n}^{j}\right|+\frac{a_{n}}{1+\lambda_{1} a_{n}}\left\|B_{n}-B\right\|\left\|\widetilde{U}_{n}\right\| .
$$

By H2b' and since $\left\|\widetilde{U}_{n}\right\|$ converges, applying Lemma 7 yields: $\left|\widetilde{U}_{n}^{j}\right| \underset{n \rightarrow+\infty}{\longrightarrow} \widetilde{U}^{j}, \sum_{n=1}^{\infty} \alpha_{n}\left|\widetilde{U}_{n}^{j}\right|<\infty$.

Since $\sum_{n=1}^{\infty} a_{n}=\infty, \widetilde{U}^{j}=0$.

(b) For $j=1$, by H2b' and since $\left\|\widetilde{U}_{n}\right\| \longrightarrow \sqrt{\widetilde{U}}$ when $n \longrightarrow \infty$ :

$$
\begin{aligned}
\widetilde{U}_{n+1}^{1} & =\widetilde{U}_{n}^{1}+\frac{a_{n}}{1+\lambda_{1} a_{n}}\left\langle V_{1},\left(B_{n}-B\right) \widetilde{U}_{n}\right\rangle=\widetilde{U}_{1}^{1}+\sum_{i=1}^{n} \frac{a_{i}}{1+\lambda_{1} a_{i}}\left\langle V_{1},\left(B_{i}-B\right) \widetilde{U}_{i}\right\rangle \\
& \longrightarrow \widetilde{U}^{1}=\widetilde{U}_{1}^{1}+\sum_{i=1}^{\infty} \frac{a_{i}}{1+\lambda_{1} a_{i}}\left\langle V_{1},\left(B_{i}-B\right) \widetilde{U}_{i}\right\rangle .
\end{aligned}
$$

Now:

$$
\widetilde{U}_{n+1}^{1}=\left\langle V_{1}, \widetilde{U}_{n+1}\right\rangle=\left\langle V_{1}, \prod_{i=1}^{n} \frac{I+a_{i} B_{i}}{1+\lambda_{1} a_{i}} \widetilde{U}_{1}\right\rangle \rightarrow\left\langle V_{1}, \prod_{i=1}^{\infty} \frac{I+a_{i} B_{i}}{1+\lambda_{1} a_{i}} \widetilde{U}_{1}\right\rangle=V_{1}^{\prime} Q S \widetilde{U}_{1}=\widetilde{U}^{1}, S=\prod_{i=1}^{\infty} \frac{I+a_{i} B_{i}}{1+\lambda_{1} a_{i}} .
$$

Since $\widetilde{U}_{1}$ is absolutely continuous, if $V_{1}^{\prime} Q S \neq 0, \operatorname{Pr}\left(V_{1}^{\prime} Q S \widetilde{U}_{1}=0 \mid S\right)=0$, then $\operatorname{Pr}\left(\widetilde{U}^{1}=0\right)=0$. Prove that $V_{1}^{\prime} Q S \neq 0$.

Step 3. Denote $C_{2}=\left\{\widetilde{U}_{1} \neq 0\right\}$. Suppose $\omega \in C_{1} \cap C_{2}$.

Under $\mathrm{H} 2 \mathrm{~b}$ ' and since $a_{n} \longrightarrow 0$, there exists $N$ such that $\sum_{n=N}^{\infty} a_{n}\left\|B_{n}-B\right\|<\ln 2$ and all eigenvalues of $I+a_{i} B$ are positive for $i \geqslant N$, then $\left\|I+a_{i} B\right\|=1+\lambda_{1} a_{i}$ and

$$
V_{1}^{\prime} Q S=V_{1}^{\prime} Q \prod_{i=N}^{\infty} \frac{I+a_{i} B_{i}}{1+\lambda_{1} a_{i}} \prod_{i=1}^{N-1} \frac{I+a_{i} B_{i}}{1+\lambda_{1} a_{i}}=V_{1}^{\prime} Q R \prod_{i=1}^{N-1} \frac{I+a_{i} B_{i}}{1+\lambda_{1} a_{i}}, R=\prod_{i=N}^{\infty} \frac{I+a_{i} B_{i}}{1+\lambda_{1} a_{i}} .
$$

Under H2c, $V_{1}^{\prime} Q S \neq 0 \Leftrightarrow V_{1}^{\prime} Q R \neq 0$. Let $C_{n}=\frac{a_{n}\left\|B_{n}-B\right\|}{1+\lambda_{1} a_{n}}$ and $\left(W_{n}, n \geqslant N\right)$ be the process $\left(\widetilde{U}_{n}, n \geqslant N\right)$ with $W_{N}=V_{1}$. By Step 2 , since $W_{N}=V_{1}$, for $i \in\{N+1, \ldots, n\}$ :

$$
\begin{aligned}
& W_{n+1}^{1}=\left\langle V_{1}, W_{n+1}\right\rangle=1+\sum_{i=N}^{n} \frac{a_{i}}{1+\lambda_{1} a_{i}}\left\langle V_{1},\left(B_{i}-B\right) W_{i}\right\rangle \geqslant 1-\sum_{i=N}^{n} C_{i}\left\|W_{i}\right\| ; \\
& \left\|W_{i}\right\| \leq \frac{\left\|I+a_{i-1} B_{i-1}\right\|}{1+\lambda_{1} a_{i-1}}\left\|W_{i-1}\right\| \leq \frac{\left\|I+a_{i-1} B\right\|+a_{i-1}\left\|B_{i-1}-B\right\|}{1+\lambda_{1} a_{i-1}}\left\|W_{i-1}\right\|=\left(1+C_{i-1}\right)\left\|W_{i-1}\right\| \leq \prod_{l=N}^{i-1}\left(1+C_{l}\right) .
\end{aligned}
$$

Since $\sum_{n=N}^{\infty} C_{n}<\ln 2$, it follows that $W_{n+1}^{1} \geqslant 1-\sum_{i=N}^{n} C_{i} \prod_{l=N}^{i-1}\left(1+C_{l}\right)=1-\left(\prod_{l=N}^{n}\left(1+C_{l}\right)-1\right) \geqslant 2-e^{\sum_{l=N}^{n} C_{l}}>0$. By Step $2, W_{n}^{1}$ converges to $\left\langle V_{1}, R V_{1}\right\rangle=V_{1}^{\prime} Q R V_{1}$ which is therefore strictly positive, thus $V_{1}^{\prime} Q R \neq 0$.

Step 4: conclusion. It follows that $\left(\widetilde{U}_{n}\right)$ converges to $\widetilde{U}^{1} V_{1} \neq 0$, therefore $\frac{\widetilde{U}_{n}}{\left\|\widetilde{U}_{n}\right\|}=X_{n}$ converges to $\pm V_{1}$, and by the conclusion of Step 1, $\sum_{n=1}^{\infty} a_{n}\left(\lambda_{1}-\left\langle X_{n}, B X_{n}\right\rangle\right)<\infty$. Moreover, by H2b':

$\sum_{n=1}^{\infty} a_{n}\left|\lambda_{1}-\left\langle X_{n}, B_{n} X_{n}\right\rangle\right|=\sum_{n=1}^{\infty} a_{n}\left|\lambda_{1}-\left\langle X_{n},\left(B_{n}-B\right) X_{n}\right\rangle-\left\langle X_{n}, B X_{n}\right\rangle\right|$

$\leq \sum_{n=1}^{\infty} a_{n}\left(\lambda_{1}-\left\langle X_{n}, B X_{n}\right\rangle\right)+\sum_{n=1}^{\infty} a_{n}\left\|B_{n}-B\right\|<\infty$.

This concludes the proof.

Remark. Step 1 can be replaced by: $\left\|\widetilde{U}_{n+1}\right\| \leq \frac{\left\|I+a_{n} B_{n}\right\|}{1+\lambda_{1} a_{n}}\left\|\widetilde{U}_{n}\right\| \leq\left(1+\frac{a_{n}\left\|B_{n}-B\right\|}{1+\lambda_{1} a_{n}}\right)\left\|\widetilde{U}_{n}\right\|$.Under H2b', $\left\|\widetilde{U}_{n}\right\|$ converges a.s. Assumption $\sum_{n=1}^{\infty} a_{n}^{2}<\infty$ is not used and can be replaced by $a_{n} \longrightarrow 0$ when $n \longrightarrow \infty$, but in this case the convergence of $\sum_{n=1}^{\infty} a_{n}\left(\lambda_{1}-\left\langle B X_{n}, X_{n}\right\rangle\right)$ and $\sum_{n=1}^{\infty} a_{n}\left|\lambda_{1}-\left\langle B_{n} X_{n}, X_{n}\right\rangle\right|$ is not proven.

Proof of Corollary 2, first part. Let us first recall some concepts of exterior algebra used in the proof. Let $\left(e_{1}, \ldots, e_{p}\right)$ be a basis of $\mathbb{R}^{p}$. For $r \leq p$, let ${ }^{r} \Lambda \mathbb{R}^{p}$ be the exterior power of order $r$ of $\mathbb{R}^{p}$, generated by the $C_{p}^{r}$ exterior products $e_{i_{1}} \wedge e_{i_{2}} \wedge \cdots \wedge e_{i_{r}}, i_{1}<i_{2}<\cdots<i_{r} \in\{1, \ldots, p\}$.

(a) Let $Q$ be a metric in $\mathbb{R}^{p}$. Define the inner product $\langle.,$.$\rangle in { }^{r} \Lambda \mathbb{R}^{p}$ induced by the metric $Q$ such that:

$$
\left\langle e_{i_{1}} \wedge \cdots \wedge e_{i_{r}}, e_{k_{1}} \wedge \cdots \wedge e_{k_{r}}\right\rangle=\sum_{\sigma \in G_{r}}(-1)^{s(\sigma)}\left\langle e_{i_{1}}, e_{\sigma\left(k_{1}\right)}\right\rangle \times \cdots \times\left\langle e_{i_{r}}, e_{\sigma\left(k_{r}\right)}\right\rangle,
$$


$G_{r}$ being the set of permutations $\sigma$ of $\left\{k_{1}, \ldots, k_{r}\right\}$ and $s(\sigma)$ the number of inversions of $\sigma$. The associated norm is also denoted $\|$.$\| . Note that if x_{1}, \ldots, x_{r}$ are $Q$-orthogonal, $\left\|x_{1} \wedge \cdots \wedge x_{r}\right\|=\prod_{i=1}^{r}\left\|x_{i}\right\|$, and if $\left(e_{1}, \ldots, e_{p}\right)$ is a $Q$-orthonormal basis of $\mathbb{R}^{p}$, then the set of the $C_{p}^{r}$ exterior products $e_{i_{1}} \wedge \cdots \wedge e_{i_{r}}$ is an orthonormal basis of ${ }^{r} \Lambda \mathbb{R}^{p}$.

(b) Let $U$ be an endomorphism in $\mathbb{R}^{p}$. Define for $j \in\{1, \ldots, r\}$ the endomorphism ${ }^{r j} U$ in ${ }^{r} \Lambda \mathbb{R}^{p}$ such that:

$$
\begin{aligned}
{ }^{r j} U\left(x_{1} \wedge \cdots \wedge x_{r}\right) & =\sum_{1 \leq i_{1}<i_{2}<\ldots<i_{j} \leq r} x_{1} \wedge \cdots \wedge U x_{i_{1}} \wedge \cdots \wedge U x_{i_{j}} \wedge \cdots \wedge x_{r} \\
\text { for } j & =1,{ }^{r 1} U\left(x_{1} \wedge \cdots \wedge x_{r}\right)=\sum_{i=1}^{r} x_{1} \wedge \cdots \wedge U x_{i} \wedge \cdots \wedge x_{r} \\
\text { for } j & =r,{ }^{r r} U\left(x_{1} \wedge \cdots \wedge x_{r}\right)=U x_{1} \wedge U x_{2} \wedge \cdots \wedge U x_{r} .
\end{aligned}
$$

(c) The following properties hold:

(i) Suppose $U$ has $p$ real eigenvalues $\lambda_{1}, \ldots, \lambda_{p}$ and, for $j \in\{1, \ldots, p\}$, let $V_{j}$ be an eigenvector corresponding to $\lambda_{j}$. Then the $C_{p}^{r}$ vectors $V_{i_{1}} \wedge \cdots \wedge V_{i_{r}}, 1 \leq i_{1}<\cdots<i_{r} \leq p$, are eigenvectors of ${ }^{r j} U$; for $j=1$, the corresponding eigenvalues are $\lambda_{i_{1}}+\cdots+\lambda_{i_{r}}$ and for $j=r$, the products $\lambda_{i_{1}} \cdots \lambda_{i_{r}}$; thus if $U$ is invertible, so is ${ }^{r r} U$;

(ii) If $U$ is $Q$-symmetric, ${ }^{r j} U$ is symmetric with respect to the metric induced by $Q$ in ${ }^{r} \Lambda \mathbb{R}^{p}$;

(iii) ${ }^{r r}(I+U)=I+\sum_{j=1}^{r}{ }^{r j} U$;

(iv) There exists $c(r)>0$ such that, for every endomorphism $U$ in $\mathbb{R}^{p}$ and for $1 \leq j \leq r,\left\|^{r j} U\right\| \leq c(r)\|U\|^{j}$.

Outline of the proof. Applying the first assertion of Theorem 1, we prove in the first step that for $i \in\{1, \ldots, r\}$, ${ }^{i} X_{n}=X_{n}^{1} \wedge \cdots \wedge X_{n}^{i}$ converges a.s. to an eigenvector $\pm V_{j_{1}} \wedge \cdots \wedge V_{j_{i}}$ of ${ }^{i 1} B$. In the second step, we prove by recurrence on $i$ that $X_{n}^{i}$ converges a.s. to $\pm V_{i}$, by proving first that there exists $j>i-1$ such that ${ }^{i} X_{n}$ converges a.s. to $\pm V_{1} \wedge \cdots \wedge V_{i-1} \wedge V_{j}$, thus $X_{n}^{i}$ converges a.s. to $\pm V_{j}$, and then, applying the second assertion of Theorem 1, that $V_{j}= \pm V_{i}$. In the third step, we prove that $\sum_{n=1}^{\infty} a_{n}\left|\lambda_{i}-\left\langle B X_{n}^{i}, X_{n}^{i}\right\rangle\right|$ and $\sum_{n=1}^{\infty} a_{n}\left(\lambda_{i}-\left\langle B_{n} X_{n}^{i}, X_{n}^{i}\right\rangle\right)$ converge a.s.

Step 1. For $i \in\{1, \ldots, r\}$, it follows from the orthogonality of $T_{n}^{1}, \ldots, T_{n}^{i}$ that $\left\|T_{n+1}^{1} \wedge \cdots \wedge T_{n+1}^{i}\right\|=\prod_{l=1}^{i}\left\|T_{n+1}^{l}\right\|$. Let ${ }^{i} X_{n+1}=X_{n+1}^{1} \wedge \cdots \wedge X_{n+1}^{i}$ and $D_{n}^{i}={ }^{i 1} B_{n}+\sum_{j=2}^{i} a_{n}^{j-1}{ }^{i j} B_{n}$. Then:

$$
\begin{aligned}
{ }^{i} X_{n+1}= & \frac{T_{n+1}^{1} \wedge \cdots \wedge T_{n+1}^{i}}{\left\|T_{n+1}^{1} \wedge \cdots \wedge T_{n+1}^{i}\right\|}=\frac{Y_{n+1}^{1} \wedge \cdots \wedge Y_{n+1}^{i}}{\left\|Y_{n+1}^{1} \wedge \cdots \wedge Y_{n+1}^{i}\right\|}=\frac{i i\left(I+a_{n} B_{n}\right){ }^{i} X_{n}}{\left\|{ }^{i i}\left(I+a_{n} B_{n}\right){ }^{i} X_{n}\right\|} \\
= & \frac{\left(I+a_{n}{ }^{i 1} B_{n}+\sum_{j=2}^{i} a_{n}^{j}{ }^{j i j} B_{n}\right){ }^{i} X_{n}}{\left\|\left(I+a_{n}{ }^{i 1} B_{n}+\sum_{j=2}^{i} a_{n}^{j}{ }^{j i} B_{n}\right){ }^{i} X_{n}\right\|}=\frac{\left(I+a_{n} D_{n}^{i}\right){ }^{i} X_{n}}{\left\|\left(I+a_{n} D_{n}^{i}\right){ }^{i} X_{n}\right\|} .
\end{aligned}
$$

Since $\left\|^{i j} B_{n}\right\| \leq c(i)\left\|B_{n}\right\|^{j}$, assumptions H2a ( $\sup _{n}\left\|B_{n}\right\|<b$ a.s.) and H3 yield that there exists $b_{1}>0$ such that for all $n,\left\|D_{n}^{i}\right\| \leq b_{1}$. Moreover, since $U \mapsto{ }^{i 1} U$ is a linear application, assumptions H2a,b $\left(\sum_{n=1}^{\infty} a_{n} E\left[\left\|E\left[B_{n} \mid T_{n}\right]-B\right\|\right]<\infty\right)$ and $\mathrm{H} 3$ yield that:

$$
\begin{aligned}
& E\left[\sum_{n=1}^{\infty} a_{n}\left\|E\left[D_{n}^{i} \mid T_{n}\right]-{ }^{i 1} B\right\|\right]=E\left[\sum_{n=1}^{\infty} a_{n}\left\|E\left[{ }^{i 1} B_{n}-{ }^{i 1} B+\sum_{j=2}^{i} a_{n}^{j-1}{ }^{i j} B_{n} \mid T_{n}\right]\right\|\right] \\
& \leq E\left[\sum_{n=1}^{\infty} a_{n}\left(\left\|{ }^{i 1} E\left[B_{n}-B \mid T_{n}\right]\right\|+\sum_{j=2}^{i} a_{n}^{j-1} E\left[\left\|^{i j} B_{n}\right\| \mid T_{n}\right]\right)\right] \\
& \left.\leq c(i) E\left[\sum_{n=1}^{\infty} a_{n}\left(\| E\left[B_{n} \mid T_{n}\right]-B\right] \|+\sum_{j=2}^{i} a_{n}^{j-1} E\left[\left\|B_{n}\right\|^{j} \mid T_{n}\right]\right)\right]<\infty .
\end{aligned}
$$

${ }^{i 1} B$ is symmetric with respect to the metric induced by $Q$ in ${ }^{i} \Lambda \mathbb{R}^{p}$ and by $\mathrm{H} 1 \mathrm{~b}$ ' its largest eigenvalue is simple. Applying the first assertion of Theorem 1 yields that almost surely:

$$
\begin{aligned}
& { }^{i} X_{n} \text { converges to a normed eigenvector } \pm V_{j_{1}} \wedge \cdots \wedge V_{j_{i}} \text { of }{ }^{i 1} B, \\
& \sum_{n=1}^{\infty} a_{n}\left(\lambda_{j_{1}}+\cdots+\lambda_{j_{i}}-\left\langle{ }^{i 1} B{ }^{i} X_{n},{ }^{i} X_{n}\right\rangle\right)<\infty, \sum_{n=1}^{\infty} a_{n}\left(\lambda_{j_{1}}+\cdots+\lambda_{j_{i}}-\left\langle D_{n}^{i}{ }^{i} X_{n},{ }^{i} X_{n}\right\rangle\right)<\infty .
\end{aligned}
$$

Moreover, by $\mathrm{H} 2 \mathrm{a}$ and $\mathrm{H} 3, \sum_{n=1}^{\infty} a_{n}\left(\lambda_{j_{1}}+\cdots+\lambda_{j_{i}}-\left\langle{ }^{i 1} B_{n}{ }^{i} X_{n},{ }^{i} X_{n}\right\rangle\right)<\infty$. 
Step 2. Suppose that for $k \in\{1, \ldots, i-1\}$, when $n \longrightarrow \infty, X_{n}^{k} \longrightarrow \pm V_{k}$, which is verified for $k=1$ by Theorem 1 , and prove that $X_{n}^{i} \longrightarrow \pm V_{i}$.

(a) Prove that there exists $j>i-1$ such that $X_{n}^{i} \longrightarrow \pm V_{j}$.

${ }^{i} X_{n} \longrightarrow \pm V_{j_{1}} \wedge \cdots \wedge V_{j_{i}}$; suppose that there exists $k \in\{1, \ldots, i-1\}$ such that, for $l \in\{1, \ldots, i\}, V_{j_{l}} \neq \pm V_{k}$; since $X_{n}^{k} \longrightarrow \pm V_{k},\left\langle X_{n}^{k}, V_{j_{l}}\right\rangle \longrightarrow 0$ for $l \in\{1, \ldots, i\}$ and $\left\langle X_{n}^{1} \Lambda \cdots \Lambda X_{n}^{i}, V_{j_{1}} \Lambda \cdots \Lambda V_{j_{i}}\right\rangle \longrightarrow 0$, a contradiction. Therefore for all $k \in\{1, \ldots, i-1\}$, there exists $j_{l}$ such that $V_{j_{l}}= \pm V_{k}$ and there exists $j$ such that ${ }^{i} X_{n} \longrightarrow \pm V_{1} \Lambda \cdots \Lambda V_{i-1} \wedge V_{j}$.

The only term which has a non-zero limit in the development of $\left\langle X_{n}^{1} \wedge \cdots \wedge X_{n}^{i}, \pm V_{1} \wedge \cdots \wedge V_{i-1} \wedge V_{j}\right\rangle$, whose limit is 1 when $n \longrightarrow \infty$, is $\pm\left\langle X_{n}^{1}, V_{1}\right\rangle\left\langle X_{n}^{2}, V_{2}\right\rangle \times \cdots \times\left\langle X_{n}^{i-1}, V_{i-1}\right\rangle\left\langle X_{n}^{i}, V_{j}\right\rangle$ obtained for $\sigma=I d$. Since for $k \in\{1, \ldots, i-1\}$, $\left\langle X_{n}^{k}, V_{k}\right\rangle \longrightarrow \pm 1$, then $\left\langle X_{n}^{i}, V_{j}\right\rangle \longrightarrow \pm 1$. Therefore, $X_{n}^{i} \longrightarrow \pm V_{j}$.

(b) Prove now that $V_{j}= \pm V_{i}$. Suppose $X_{n}^{i} \longrightarrow \pm V_{j} \neq \pm V_{i}$.

Let $G_{i}$ be the set of permutations $\sigma$ of $\{1, \ldots, i\}$ with $\sigma=(\sigma(1), \ldots, \sigma(i))$ and $s(\sigma)$ the number of inversions of $\sigma$. Prove that $\lim \inf E\left[\left\langle D_{n}^{i}\left(X_{n}^{1} \wedge \cdots \wedge X_{n}^{i}\right), V_{1} \wedge \cdots \wedge V_{i}\right\rangle^{2} \mid T_{n}\right]>0, D_{n}^{i}={ }^{i 1} B_{n}+\sum_{j=2}^{i} a_{n}^{j-1 i j} B_{n}$.

$$
\begin{aligned}
\left\langle{ }^{i 1} B_{n}\left(X_{n}^{1} \wedge \cdots \wedge X_{n}^{i}\right), V_{1} \wedge \cdots \wedge V_{i}\right\rangle & =\sum_{l=1}^{i}\left\langle X_{n}^{1} \wedge \cdots \wedge B_{n} X_{n}^{l} \wedge \cdots \wedge X_{n}^{i}, V_{1} \wedge \cdots \wedge V_{i}\right\rangle \\
& =\sum_{l=1}^{i} \sum_{\sigma \in G_{i}}(-1)^{s(\sigma)}\left\langle X_{n}^{1}, V_{\sigma(1)}\right\rangle \times \cdots \times\left\langle B_{n} X_{n}^{l}, V_{\sigma(l)}\right\rangle \times \cdots \times\left\langle X_{n}^{i}, V_{\sigma(i)}\right\rangle, \\
E\left[\left\langle{ }^{i 1} B_{n}\left(X_{n}^{1} \wedge \cdots \wedge X_{n}^{i}\right), V_{1} \wedge \cdots \wedge V_{i}\right\rangle^{2} \mid T_{n}\right] & =E\left[\left(\sum_{l=1}^{i} \sum_{\sigma \in G_{i}}(-1)^{s(\sigma)}\left\langle X_{n}^{1}, V_{\sigma(1)}\right\rangle \cdots\left\langle B_{n} X_{n}^{l}, V_{\sigma(l)}\right\rangle \cdots\left\langle X_{n}^{i}, V_{\sigma(i)}\right\rangle\right)^{2} \mid T_{n}\right] .
\end{aligned}
$$

Since for $k \in\{1, \ldots, i-1\}, X_{n}^{k} \longrightarrow \pm V_{k}$, the only term with a non-zero limit in the development of this conditional expectation is $\left\langle X_{n}^{1}, V_{1}\right\rangle^{2} \times \cdots \times\left\langle X_{n}^{i-1}, V_{i-1}\right\rangle^{2} E\left[\left\langle B_{n} X_{n}^{i}, V_{i}\right\rangle^{2} \mid T_{n}\right]$ and

$$
\liminf E\left[\left\langle{ }^{i 1} B_{n}\left(X_{n}^{1} \wedge \cdots \wedge X_{n}^{i}\right), V_{1} \wedge \cdots \wedge V_{i}\right\rangle^{2} \mid T_{n}\right]=\liminf E\left[\left\langle B_{n} X_{n}^{i}, V_{i}\right\rangle^{2} \mid T_{n}\right]>0 .
$$

Moreover, by H2a and $\lim _{n \longrightarrow \infty} a_{n}=0, \liminf E\left[\left\langle\sum_{j=2}^{i} a_{n}^{j-1 i j} B_{n}\left(X_{n}^{1} \wedge \cdots \wedge X_{n}^{i}\right), V_{1} \wedge \cdots \wedge V_{i}\right\rangle^{2} \mid T_{n}\right]=0$.

Then $\lim \inf E\left[\left\langle D_{n}^{i}\left(X_{n}^{1} \wedge \cdots \wedge X_{n}^{i}\right), V_{1} \wedge \cdots \wedge V_{i}\right\rangle^{2} \mid T_{n}\right]>0$. Applying the second assertion of Theorem 1 yields a.s.:

$$
\begin{aligned}
& X_{n}^{1} \wedge \cdots \wedge X_{n}^{i} \longrightarrow \pm V_{1} \wedge \cdots \wedge V_{i}, \text { therefore } X_{n}^{i} \longrightarrow \pm V_{i}, \\
& \sum_{n=1}^{\infty} a_{n}\left(\sum_{l=1}^{i} \lambda_{l}-\left\langle{ }^{i 1} B{ }^{i} X_{n},{ }^{i} X_{n}\right\rangle\right)<\infty, \sum_{n=1}^{\infty} a_{n}\left(\sum_{l=1}^{i} \lambda_{l}-\left\langle D_{n}^{i}{ }^{i} X_{n},{ }^{i} X_{n}\right\rangle\right)<\infty .
\end{aligned}
$$

Then, by H2a and $\mathrm{H} 3, \sum_{n=1}^{\infty} a_{n}\left(\sum_{l=1}^{i} \lambda_{l}-\left\langle{ }^{i 1} B_{n}{ }^{i} X_{n},{ }^{i} X_{n}\right\rangle\right)<\infty$.

Step 3. Since $X_{n}^{1}, \ldots, X_{n}^{i}$ are orthonormal, $\left\langle{ }^{i 1} B^{i} X_{n},{ }^{i} X_{n}\right\rangle=\sum_{k=1}^{i} \sum_{\sigma \in G_{i}}(-1)^{s(\sigma)}\left\langle X_{n}^{1}, X_{n}^{\sigma(1)}\right\rangle \times \cdots \times\left\langle B X_{n}^{k}, X_{n}^{\sigma(k)}\right\rangle \times \cdots \times$ $\left\langle X_{n}^{i}, X_{n}^{\sigma(i)}\right\rangle=\sum_{k=1}^{i}\left\langle X_{n}^{1}, X_{n}^{1}\right\rangle \times \cdots \times\left\langle B X_{n}^{k}, X_{n}^{k}\right\rangle \times \cdots \times\left\langle X_{n}^{i}, X_{n}^{i}\right\rangle=\sum_{k=1}^{i}\left\langle B X_{n}^{k}, X_{n}^{k}\right\rangle$. Then, since $\sum_{l=1}^{i} \lambda_{l}$ is the largest eigenvalue of ${ }^{i 1} B$ :

$$
\begin{aligned}
& \lambda_{i}-\left\langle B X_{n}^{i}, X_{n}^{i}\right\rangle=\left(\sum_{l=1}^{i} \lambda_{l}-\left\langle{ }^{i 1} B{ }^{i} X_{n},{ }^{i} X_{n}\right\rangle\right)-\left(\sum_{l=1}^{i-1} \lambda_{l}-\left\langle{ }^{i-1,1} B{ }^{i-1} X_{n},{ }^{i-1} X_{n}\right\rangle\right) \\
& \left|\lambda_{i}-\left\langle B X_{n}^{i}, X_{n}^{i}\right\rangle\right| \leq\left(\sum_{l=1}^{i} \lambda_{l}-\left\langle{ }^{i 1} B{ }^{i} X_{n},{ }^{i} X_{n}\right\rangle\right)+\left(\sum_{l=1}^{i-1} \lambda_{l}-\left\langle{ }^{i-1,1} B^{i-1} X_{n},{ }^{i-1} X_{n}\right\rangle\right) .
\end{aligned}
$$

Since $\sum_{n=1}^{\infty} a_{n}\left(\sum_{l=1}^{i} \lambda_{l}-\left\langle{ }^{i 1} B^{i} X_{n},{ }^{i} X_{n}\right\rangle\right)<\infty$ a.s., then $\sum_{n=1}^{\infty} a_{n}\left|\lambda_{i}-\left\langle B X_{n}^{i}, X_{n}^{i}\right\rangle\right|<\infty$ a.s. Likewise: since $\sum_{n=1}^{\infty} a_{n}\left(\sum_{l=1}^{i} \lambda_{l}-\left\langle{ }^{i 1} B_{n}{ }^{i} X_{n},{ }^{i} X_{n}\right\rangle\right)<\infty$, then $\sum_{n=1}^{\infty} a_{n}\left(\lambda_{i}-\left\langle B_{n} X_{n}^{i}, X_{n}^{i}\right\rangle\right)<\infty$ a.s.

Proof of Corollary 2, second part. We prove applying Theorem 1 that ${ }^{i} X_{n}=X_{n}^{1} \wedge \cdots \wedge X_{n}^{i}$ converges a.s. to 
$\pm V_{1} \wedge \cdots \wedge V_{i}$, then by recurrence on $i$ that $X_{n}^{i}$ converges a.s. to $\pm V_{i}$.

Let $\omega$ be fixed throughout the proof, belonging to the intersection of the a.s. convergence sets. Its writing will be omitted. Let $i \in\{1, \ldots, r\}$. As already seen in the proof of Corollary 2 , first part:

$$
{ }^{i} X_{n+1}=\frac{\left(I+a_{n} D_{n}^{i}\right){ }^{i} X_{n}}{\left\|\left(I+a_{n} D_{n}^{i}\right){ }^{i} X_{n}\right\|}, D_{n}^{i}={ }^{i 1} B_{n}+\sum_{j=2}^{i} a_{n}^{j-1}{ }^{i j} B_{n} .
$$

By $\mathrm{H} 2 \mathrm{~b}^{\prime}$ and $\mathrm{H} 3$ :

$$
\sum_{n=1}^{\infty} a_{n}\left\|D_{n}^{i}-{ }^{i 1} B\right\|=\sum_{n=1}^{\infty} a_{n}\|\|^{i 1}\left(B_{n}-B\right)+\sum_{j=2}^{i} a_{n}^{j-1}{ }^{i j} B_{n} \| \leq c(i)\left(\sum_{n=1}^{\infty} a_{n}\left\|B_{n}-B\right\|+\sum_{j=2}^{i} \sum_{n=1}^{\infty} a_{n}^{j}\left\|B_{n}\right\|^{j}\right)<\infty .
$$

By $\mathrm{H} 2 \mathrm{c}, I+a_{n} B_{n}$ has no null eigenvalue, then ${ }^{i i}\left(I+a_{n} B_{n}\right)$ has no null eigenvalue and is invertible.

Since $B$ is $Q$-symmetric with distinct eigenvalues (H1a,b'), ${ }^{i 1} B$ is symmetric with respect to the metric induced by $Q$ in ${ }^{i} \Lambda \mathbb{R}^{p}$ and its largest eigenvalue $\lambda_{1}+\cdots+\lambda_{i}$ is simple; $V_{1} \wedge \cdots \wedge V_{i}$ is a normed eigenvector corresponding to this eigenvalue. Applying the second part of Theorem 1 yields that:

$$
{ }^{i} X_{n} \longrightarrow \pm V_{1} \wedge \cdots \wedge V_{i}, \sum_{n=1}^{\infty} a_{n}\left(\sum_{l=1}^{i} \lambda_{l}-\left\langle{ }^{i 1} B{ }^{i} X_{n},{ }^{i} X_{n}\right\rangle\right)<\infty, \sum_{n=1}^{\infty} a_{n}\left|\sum_{l=1}^{i} \lambda_{l}-\left\langle D_{n}^{i i} X_{n},{ }^{i} X_{n}\right\rangle\right|<\infty .
$$

It implies that $\sum_{n=1}^{\infty} a_{n}\left|\sum_{l=1}^{i} \lambda_{l}-\left\langle{ }^{i 1} B_{n}{ }^{i} X_{n},{ }^{i} X_{n}\right\rangle\right|<\infty$ since $D_{n}^{i}={ }^{i 1} B_{n}+\sum_{j=2}^{i} a_{n}^{j-1}{ }^{i j} B_{n}$ and

$$
\sum_{n=1}^{\infty} a_{n}\left|\left\langle\sum_{j=2}^{i} a_{n}^{j-1}{ }^{i j} B_{n}{ }^{i} X_{n},{ }^{i} X_{n}\right\rangle\right| \leq \sum_{j=2}^{i} \sum_{n=1}^{\infty} a_{n}^{j}\left\|^{i j} B_{n}\right\| \leq c(i) \sum_{j=2}^{i} \sum_{n=1}^{\infty} a_{n}^{j}\left\|B_{n}\right\|^{j} \leq c(i) \sum_{j=2}^{i} 2^{j-1} \sum_{n=1}^{\infty} a_{n}^{j}\left(\left\|B_{n}-B\right\|^{j}+\|B\|^{j}\right)<\infty .
$$

Suppose that, for $k \in\{1, \ldots, i-1\}, X_{n}^{k}$ converges to $\pm V_{k}$, which is verified for $k=1$ by Theorem 1 , and prove that it is true for $k=i$. In the development of $\left\langle X_{n}^{1} \wedge \cdots \wedge X_{n}^{i}, \pm V_{1} \wedge \cdots \wedge V_{i}\right\rangle$, which converges to \pm 1 , the only term which has a non-zero limit is $\left\langle X_{n}^{1}, V_{1}\right\rangle \times \cdots \times\left\langle X_{n}^{i-1}, V_{i-1}\right\rangle\left\langle X_{n}^{i}, V_{i}\right\rangle$; since for $k \in\{1, \ldots, i-1\},\left\langle X_{n}^{k}, V_{k}\right\rangle$ converges to \pm 1 , it follows that $\left\langle X_{n}^{i}, V_{i}\right\rangle$ converges to \pm 1 , thus $X_{n}^{i}$ converges to $\pm V_{i}$.

Applying the same argument as in the previous proof, Step 3, yields: $\sum_{n=1}^{\infty} a_{n}\left|\lambda_{i}-\left\langle X_{n}^{i}, B X_{n}^{i}\right\rangle\right|<\infty$. By H2b':

$$
\sum_{n=1}^{\infty} a_{n}\left|\lambda_{i}-\left\langle X_{n}^{i}, B_{n} X_{n}^{i}\right\rangle\right| \leqslant \sum_{n=1}^{\infty} a_{n}\left|\lambda_{i}-\left\langle X_{n}^{i}, B X_{n}^{i}\right\rangle\right|+\sum_{n=1}^{\infty} a_{n}\left\|B_{n}-B\right\|<\infty .
$$

Proof of Corollary 3. We verify the assumptions of the first part of Corollary 2. $B$ is symmetric (H1a), $\sup _{n}\left\|B_{n}\right\|$ is a.s. bounded under $\mathrm{H} 6 \mathrm{a}$ and $\mathrm{H} 7 \mathrm{a}$; we have almost surely:

$$
\begin{aligned}
& E\left[B_{n} \mid T_{n}\right]-B=E\left[M_{n-1}^{\frac{1}{2}}\left(\frac{1}{m_{n}} \sum_{i=1}^{m_{n}} Z_{n i}^{c}\left(Z_{n i}^{c}\right)^{\top}-\bar{Z}_{n-1}^{c}\left(\bar{Z}_{n-1}^{c}\right)^{\top}\right) M_{n-1}^{\frac{1}{2}} \mid T_{n}\right]-M^{\frac{1}{2}}\left(E\left[Z^{c}\left(Z^{c}\right)^{\top}\right]-E\left[Z^{c}\right] E\left[Z^{c}\right]^{\top}\right) M^{\frac{1}{2}} \\
& =M_{n-1}^{\frac{1}{2}}\left(E\left[Z^{c}\left(Z^{c}\right)^{\top}\right]-\bar{Z}_{n-1}^{c}\left(\bar{Z}_{n-1}^{c}\right)^{\top}\right) M_{n-1}^{\frac{1}{2}}-M^{\frac{1}{2}}\left(E\left[Z^{c}\left(Z^{c}\right)^{\top}\right]-E\left[Z^{c}\right] E\left[Z^{c}\right]^{\top}\right) M^{\frac{1}{2}} \\
& =\left(M_{n-1}^{\frac{1}{2}}-M^{\frac{1}{2}}\right)\left(E\left[Z^{c}\left(Z^{c}\right)^{\top}\right]-E\left[Z^{c}\right] E\left[Z^{c}\right]^{\top}\right) M_{n-1}^{\frac{1}{2}}+M^{\frac{1}{2}}\left(E\left[Z^{c}\left(Z^{c}\right)^{\top}\right]-E\left[Z^{c}\right] E\left[Z^{c}\right]^{\top}\right)\left(M_{n-1}^{\frac{1}{2}}-M^{\frac{1}{2}}\right) \\
& -M_{n-1}^{\frac{1}{2}}\left(\bar{Z}_{n-1}^{c}-E\left[Z^{c}\right]\right)\left(\bar{Z}_{n-1}^{c}\right)^{\top} M_{n-1}^{\frac{1}{2}}-M_{n-1}^{\frac{1}{2}} E\left[Z^{c}\right]\left(\bar{Z}_{n-1}^{c}-E\left[Z^{c}\right]\right)^{\top} M_{n-1}^{\frac{1}{2}} .
\end{aligned}
$$

If $Z$ has $4^{\text {th }}$ order moments and $a_{n}>0, \sum_{n=1}^{\infty} \frac{a_{n}}{\sqrt{n}}<\infty: \sum_{n=1}^{\infty} a_{n} E\left[\left\|\bar{Z}_{n-1}^{c}-E\left[Z^{c}\right]\right\|\right]=\sum_{n=1}^{\infty} a_{n} E\left[\left\|\bar{Z}_{n-1}-E[Z]\right\|\right]<\infty$. Therefore, under H6a, H7a,c, $E\left[\sum_{n=1}^{\infty} a_{n}\left\|E\left[B_{n} \mid T_{n}\right]-B\right\|\right]<\infty$ (H2b). By Corollary 2 , for $k=1, \ldots, r, X_{n}^{k}$ converges a.s. to one of the eigenvectors of $B$.

Prove now that $\lim _{n \rightarrow \infty} E\left[\left(\left(X_{n}^{k}\right)^{\top} B_{n} V_{k}\right)^{2} \mid T_{n}\right]>0$ a.s. on the set $\left\{X_{n}^{k} \longrightarrow V_{j}\right\}$ for $j \neq k$. In the remainder of the proof, $X_{n}^{k}$ is denoted $X_{n}$. Decompose $E\left[\left(X_{n}^{\top} B_{n} V_{k}\right)^{2} \mid T_{n}\right]$ into the sum of three terms:

$$
E\left[\left(X_{n}^{\top} M_{n-1}^{\frac{1}{2}}\left(\frac{1}{m_{n}} \sum_{i=1}^{m_{n}} Z_{n i}^{c}\left(Z_{n i}^{c}\right)^{\top}-\bar{Z}_{n-1}^{c}\left(\bar{Z}_{n-1}^{c}\right)^{\top}\right) M_{n-1}^{\frac{1}{2}} V_{k}\right)^{2} \mid T_{n}\right]
$$




$$
\begin{aligned}
& =E\left[\left(\frac{1}{m_{n}} \sum_{i=1}^{m_{n}}\left(X_{n}^{\top} M_{n-1}^{\frac{1}{2}} Z_{n i}^{c}\right)\left(\left(Z_{n i}^{c}\right)^{\top} M_{n-1}^{\frac{1}{2}} V_{k}\right)-\left(X_{n}^{\top} M_{n-1}^{\frac{1}{2}} \bar{Z}_{n-1}^{c}\right)\left(\left(\bar{Z}_{n-1}^{c}\right)^{\top} M_{n-1}^{\frac{1}{2}} V_{k}\right)\right)^{2} \mid T_{n}\right]=A_{n}+B_{n}+C_{n}, \\
& A_{n}=E\left[\left(\frac{1}{m_{n}} \sum_{i=1}^{m_{n}}\left(X_{n}^{\top} M_{n-1}^{\frac{1}{2}} Z_{n i}^{c}\right)\left(\left(Z_{n i}^{c}\right)^{\top} M_{n-1}^{\frac{1}{2}} V_{k}\right)\right)^{2} \mid T_{n}\right], \\
& B_{n}=-2\left(X_{n}^{\top} M_{n-1}^{\frac{1}{2}} \bar{Z}_{n-1}^{c}\right)\left(\left(\bar{Z}_{n-1}^{c}\right)^{\top} M_{n-1}^{\frac{1}{2}} V_{k}\right) \frac{1}{m_{n}} \sum_{i=1}^{m_{n}} E\left[\left(X_{n}^{\top} M_{n-1}^{\frac{1}{2}} Z_{n i}^{c}\right)\left(\left(Z_{n i}^{c}\right)^{\top} M_{n-1}^{\frac{1}{2}} V_{k}\right) \mid T_{n}\right], \\
& C_{n}=\left(X_{n}^{\top} M_{n-1}^{\frac{1}{2}} \bar{Z}_{n-1}^{c}\right)^{2}\left(\left(\bar{Z}_{n-1}^{c}\right)^{\top} M_{n-1}^{\frac{1}{2}} V_{k}\right)^{2} .
\end{aligned}
$$

Note that the two random variables $R=V_{j}^{\top} M^{\frac{1}{2}} Z^{c}$ and $S=V_{k}^{\top} M^{\frac{1}{2}} Z^{c}$ are uncorrelated:

$$
\begin{aligned}
E[(R-E[R])(S-E[S])] & =E\left[V_{j}^{\top} M^{\frac{1}{2}}(Z-E[Z]) \cdot V_{k}^{\top} M^{\frac{1}{2}}(Z-E[Z])\right] \\
& =V_{j}^{\top} M^{\frac{1}{2}} E\left[(Z-E[Z])(Z-E[Z])^{\top}\right] M^{\frac{1}{2}} V_{k}=\lambda_{k} V_{j}^{\top} V_{k}=0 .
\end{aligned}
$$

Then, $E\left[V_{j}^{\top} M^{\frac{1}{2}} Z^{c} \cdot V_{k}^{\top} M^{\frac{1}{2}} Z^{c}\right]=E\left[V_{j}^{\top} M^{\frac{1}{2}} Z^{c}\right] E\left[V_{k}^{\top} M^{\frac{1}{2}} Z^{c}\right]$. Under H7b, almost surely, when $n \longrightarrow \infty$, we have:

$$
\begin{aligned}
& A_{n}=\frac{1}{m_{n}^{2}} \sum_{i=1}^{m_{n}} \sum_{l=1}^{m_{n}} E\left[\left(X_{n}^{\top} M_{n-1}^{\frac{1}{2}} Z_{n i}^{c}\right)\left(\left(Z_{n i}^{c}\right)^{\top} M_{n-1}^{\frac{1}{2}} V_{k}\right)\left(X_{n}^{\top} M_{n-1}^{\frac{1}{2}} Z_{n l}^{c}\right)\left(\left(Z_{n l}^{c}\right)^{\top} M_{n-1}^{\frac{1}{2}} V_{k}\right) \mid T_{n}\right] \\
& =X_{n}^{\top} M_{n-1}^{\frac{1}{2}} \frac{1}{m_{n}^{2}} \sum_{i=1}^{m_{n}} \sum_{l=1}^{m_{n}} E\left[\left(V_{k}^{\top} M_{n-1}^{\frac{1}{2}} Z_{n i}^{c}\right) Z_{n i}^{c}\left(Z_{n l}^{c}\right){ }^{\top}\left(\left(Z_{n l}^{c}\right)^{\top} M_{n-1}^{\frac{1}{2}} V_{k}\right) \mid T_{n}\right] M_{n-1}^{\frac{1}{2}} X_{n} \\
& \longrightarrow V_{j}^{\top} M^{\frac{1}{2}} E\left[\left(V_{k}^{\top} M^{\frac{1}{2}} Z^{c}\right) Z^{c}\left(Z^{c}\right)^{\top}\left(\left(Z^{c}\right)^{\top} M^{\frac{1}{2}} V_{k}\right)\right] M^{\frac{1}{2}} V_{j}=E\left[\left(V_{k}^{\top} M^{\frac{1}{2}} Z^{c}\right)^{2}\left(V_{j}^{\top} M^{\frac{1}{2}} Z^{c}\right)^{2}\right] \\
& B_{n} \longrightarrow-2 E\left[V_{j}^{\top} M^{\frac{1}{2}} Z^{c}\right] E\left[\left(Z^{c}\right)^{\top} M^{\frac{1}{2}} V_{k}\right] E\left[\left(V_{j}^{\top} M^{\frac{1}{2}} Z^{c}\right)\left(\left(Z^{c}\right)^{\top} M^{\frac{1}{2}} V_{k}\right)\right]=-2 E\left[\left(V_{j}^{\top} M^{\frac{1}{2}} Z^{c}\right)\left(V_{k}^{\top} M^{\frac{1}{2}} Z^{c}\right)\right]^{2} ; \\
& C_{n} \longrightarrow\left(E\left[V_{j}^{\top} M^{\frac{1}{2}} Z^{c}\right] E\left[V_{k}^{\prime} M^{\frac{1}{2}} Z^{c}\right]\right)^{2}=E\left[\left(V_{j}^{\top} M^{\frac{1}{2}} Z^{c}\right)\left(V_{k}^{\top} M^{\frac{1}{2}} Z^{c}\right)\right]^{2} ; \\
& E\left[\left(X_{n}^{\top} B_{n} V_{k}\right)^{2} \mid T_{n}\right]=A_{n}+B_{n}+C_{n} \longrightarrow E\left[\left(V_{j}^{\top} M^{\frac{1}{2}} Z^{c}\right)^{2}\left(V_{k}^{\top} M^{\frac{1}{2}} Z^{c}\right)^{2}\right]-E\left[\left(V_{j}^{\top} M^{\frac{1}{2}} Z^{c}\right)\left(V_{k}^{\top} M^{\frac{1}{2}} Z^{c}\right)\right]^{2} \\
& =\operatorname{Var}\left[V_{j}^{\top} M^{\frac{1}{2}} Z^{c} \cdot V_{k}^{\top} M^{\frac{1}{2}} Z^{c}\right]>0 \text { a.s. by H6b. }
\end{aligned}
$$

Proof of Corollary 4. Let us verify the assumptions of the second part of Corollary 2. H2c is verified since the eigenvalues of $B_{n}$ are non-negative. Prove now that $\sum_{n=1}^{\infty} a_{n}\left\|B_{n}-B\right\|<\infty$ a.s.

$$
\begin{aligned}
& B=M^{\frac{1}{2}} C M^{\frac{1}{2}}, C=E\left[Z Z^{\top}\right]-E[Z] E[Z]^{\top} ; \\
& B_{n}=M_{n}^{\frac{1}{2}} C_{n} M_{n}^{\frac{1}{2}}, C_{n}=\frac{1}{\sum_{i=1}^{n} m_{i}} \sum_{i=1}^{n} \sum_{j=1}^{m_{i}} Z_{i j}^{c}\left(Z_{i j}^{c}\right)^{\top}-\bar{Z}_{n}^{c}\left(\bar{Z}_{n}^{c}\right)^{\top}=\frac{1}{\sum_{i=1}^{n} m_{i}} \sum_{i=1}^{n} \sum_{j=1}^{m_{i}} Z_{i j}\left(Z_{i j}\right)^{\top}-\bar{Z}_{n}\left(\bar{Z}_{n}\right)^{\top} ; \\
& C_{n}-C=\frac{1}{\sum_{i=1}^{n} m_{i}} \sum_{i=1}^{n} \sum_{j=1}^{m_{i}} Z_{i j}\left(Z_{i j}\right)^{\top}-E\left[Z Z^{\top}\right]-\left(\bar{Z}_{n}-E[Z]\right)\left(\bar{Z}_{n}\right)^{\top}-E[Z]\left(\bar{Z}_{n}-E[Z]\right)^{\top} ; \\
& B_{n}-B=M_{n}^{\frac{1}{2}} C_{n} M_{n}^{\frac{1}{2}}-M^{\frac{1}{2}} C M^{\frac{1}{2}}=\left(M_{n}^{\frac{1}{2}}-M^{\frac{1}{2}}\right) C_{n} M_{n}^{\frac{1}{2}}+M^{\frac{1}{2}}\left(C_{n}-C\right) M_{n}^{\frac{1}{2}}+M^{\frac{1}{2}} C\left(M_{n}^{\frac{1}{2}}-M^{\frac{1}{2}}\right) .
\end{aligned}
$$

Under H3' and H6c, $\sum_{n=1}^{\infty} a_{n}\left\|\bar{Z}_{n}-E[Z]\right\|<\infty, \sum_{n=1}^{\infty} a_{n}\left\|\frac{1}{\sum_{i=1}^{n} m_{i}} \sum_{i=1}^{n} \sum_{j=1}^{m_{i}} Z_{i j}\left(Z_{i j}\right)^{\top}-E\left[Z Z^{\top}\right]\right\|<\infty$.

Therefore, under $\mathrm{H} 6 \mathrm{c}$ and $\mathrm{H} 7 \mathrm{~b}, \mathrm{~d}, \sum_{n=1}^{\infty} a_{n}\left\|B_{n}-B\right\|<\infty$.

\section{Acknowledgments}

We thank the Editor, Associate Editor and referees for their valuable comments and suggestions which have helped us improve the presentation of the manuscript. Results incorporated in this article received funding from the investments for the Future programme under grant agreement No ANR-15-RHU-0004.

\section{Appendix. Numerical results}

\section{Experiments}

\section{Two types of data:}

- Simulated data: At each iteration a batch of observations having a multivariate normal distribution are simulated.

- Fixed datasets: At each iteration a random sample of lines is drawn from the dataset.

\section{Algorithms: Two types}

- Batch 'B': a mini-batch of observations is used at each iteration.

- All observations 'A': all observations up to the current iteration are used. 
Algorithms are also specified by batch size. For example, Algorithm 'A_50' is the algorithm using all observations with a batch size of 50 at each iteration.

For each dataset and each algorithm, we chose either to approximate $r=3$ or $r=5$ principal components.

Algorithms initialization: The main process is initialized by choosing $X_{1}^{1}, \cdots, X_{1}^{r}$ to be a realization of a standard multivariate normal distribution.

Stepsize: $a_{n}=\frac{20}{n+1}$ where $n$ is the iteration index of the algorithm.

\section{Stopping criteria:}

- A maximum number of iterations $N=500000$.

- For $i=1, \cdots, r$, the cosine between each $X_{n}^{i}$ and the true principal factor $V^{i}$ is greater or equal 0.99 .

\section{Results:}

- For each dataset (simulated or fixed) and each algorithm, in the case of convergence, the needed computing time $t$ (in seconds) and the needed number of iterations $n$ to convergence are reported.

- For each dataset (simulated or fixed), the ranks of the algorithms regarding their needed computing time or their needed number of iterations before convergence are reported.

- When an algorithm does not converge after $N=500000$ iterations, both the needed computing time and number of iterations to convergence are considered to be "Inf".

\section{Simulated data}

$Z \in \mathbb{R}^{p}$ is simulated following multivariate normal law $\mathcal{N}(\mu, \Sigma)$ with different $p: p=50,100,500$ and two choices of $\Sigma$ referred to as Simu1 and Simu2, respectively:

- a deterministic choice: $\Sigma=A^{\top} A$ with

$$
A=\left(\begin{array}{ccccc}
\log (2) & \log (2) & \ldots & \ldots & \log (2) \\
& \log (3) & \ldots & \ldots & \log (3) \\
& & \ddots & & \\
& & & \ddots & \\
& & & & \log (p+1)
\end{array}\right)
$$

$A$ with this form was chosen since it has a natural incremental form and the log has been applied to its elements such that $\Sigma$ will not have big values.

- $\Sigma$ positive and definite matrix randomly generated using the make_sparse_spd_matrix method of the sklearn.datasets package in Python.

The following results were obtained:

\section{Needed computing time before convergence}

\begin{tabular}{|c|c|c|c|c|c|c|c|c|c|c|c|c|}
\hline Algo & $\begin{array}{l}\text { Simu1 } \\
p=50 \\
r=3\end{array}$ & $\begin{array}{l}\text { Simu1 } \\
p=50 \\
r=5\end{array}$ & $\begin{array}{l}\text { Simu1 } \\
p=100 \\
r=3\end{array}$ & $\begin{array}{l}\text { Simu1 } \\
p=100 \\
r=5\end{array}$ & $\begin{array}{l}\text { Simu1 } \\
p=500 \\
r=3\end{array}$ & $\begin{array}{l}\text { Simu1 } \\
p=500 \\
r=5\end{array}$ & $\begin{array}{l}\text { Simu2 } \\
p=50 \\
r=3\end{array}$ & $\begin{array}{l}\text { Simu2 } \\
p=50 \\
r=5\end{array}$ & $\begin{array}{l}\text { Simu2 } \\
p=100 \\
r=3\end{array}$ & $\begin{array}{l}\text { Simu2 } \\
p=100 \\
r=5\end{array}$ & $\begin{array}{l}\text { Simu2 } \\
p=500 \\
r=3\end{array}$ & $\begin{array}{l}\text { Simu2 } \\
p=500 \\
r=5\end{array}$ \\
\hline B_1 & 1.07 & 1.5 & 2.59 & 3.96 & 352.01 & 496.72 & 24.27 & Inf & 49.93 & 85.31 & 5146.88 & 5991.51 \\
\hline B_10 & 0.16 & 0.31 & 0.52 & 0.52 & 76.83 & 93.44 & 3.53 & Inf & 8.58 & 13.09 & 1356.23 & 1550.46 \\
\hline B_20 & 0.11 & 0.22 & 0.19 & 0.45 & 42.58 & 87.03 & 2.43 & 387.33 & 6.51 & 9.03 & 1107.07 & 1257.54 \\
\hline B_30 & 0.05 & 0.06 & 0.33 & 0.5 & 39.31 & 79.28 & 2.02 & 319.07 & 10.03 & 13.06 & 1100.27 & 1172.56 \\
\hline B_40 & 0.05 & 0.06 & 0.34 & 0.5 & 25.21 & 73.27 & 1.34 & 261.82 & 8.75 & 11.53 & 993.1 & 1170.65 \\
\hline B_50 & 0.05 & 0.05 & 0.3 & 0.5 & 19.81 & 73.02 & 1.27 & 241.09 & 8.34 & 11.04 & 936.33 & 1150.79 \\
\hline A_1 & 0.11 & 1.11 & 0.15 & 0.83 & 3.07 & 25.03 & 1.64 & Inf & 5.55 & 31.85 & 337.08 & 1224.85 \\
\hline A_10 & 0.02 & 0.2 & 0.02 & 0.12 & 1.02 & 6.06 & 0.27 & 207.7 & 0.94 & 4.87 & 79.08 & 284.03 \\
\hline A_20 & 0.02 & 0.07 & 0.02 & 0.08 & 0.74 & 4.87 & 0.16 & 129.7 & 0.75 & 3.44 & 67.74 & 238.63 \\
\hline A_30 & 0.02 & 0.08 & 0.02 & 0.11 & 0.88 & 4.65 & 0.16 & 106.43 & 0.94 & 5.08 & 61.68 & 219.58 \\
\hline A_40 & 0.02 & 0.08 & 0.03 & 0.12 & 1.05 & 4.46 & 0.12 & 93.12 & 1.06 & 4.39 & 55.42 & 203.94 \\
\hline A_50 & 0.0 & 0.08 & 0.02 & 0.12 & 1.1 & 1.46 & 0.12 & 85.5 & 0.72 & 4.08 & 52.6 & 199.8 \\
\hline
\end{tabular}


Needed number of iterations before convergence

\begin{tabular}{|l|l|l|l|l|l|l|l|l|l|l|l|l|}
\hline Algo & $\begin{array}{l}\text { Simu1 } \\
\mathbf{p = 5 0} \\
\mathbf{r = 3}\end{array}$ & $\begin{array}{l}\text { Simu1 } \\
\mathbf{p = 5 0} \\
\mathbf{r = 5}\end{array}$ & $\begin{array}{l}\text { Simu1 } \\
\mathbf{p = 1 0 0} \\
\mathbf{r = 3}\end{array}$ & $\begin{array}{l}\text { Simu1 } \\
\mathbf{p = 1 0 0} \\
\mathbf{r = 5}\end{array}$ & $\begin{array}{l}\text { Simu1 } \\
\mathbf{p = 5 0 0} \\
\mathbf{r = 3}\end{array}$ & $\begin{array}{l}\text { Simu1 } \\
\mathbf{p = 5 0 0} \\
\mathbf{r = 5}\end{array}$ & $\begin{array}{l}\text { Simu2 } \\
\mathbf{p = 5 0} \\
\mathbf{r = 3}\end{array}$ & $\begin{array}{l}\text { Simu2 } \\
\mathbf{p = 5 0} \\
\mathbf{r = 5}\end{array}$ & $\begin{array}{l}\text { Simu2 } \\
\mathbf{p = 1 0 0} \\
\mathbf{r = 3}\end{array}$ & $\begin{array}{l}\text { Simu2 } \\
\mathbf{p = 1 0 0} \\
\mathbf{r = 5}\end{array}$ & $\begin{array}{l}\text { Simu2 } \\
\mathbf{p = 5 0 0} \\
\mathbf{r = 3}\end{array}$ & $\begin{array}{l}\text { Simu2 } \\
\mathbf{p = 5 0 0} \\
\mathbf{r = 5}\end{array}$ \\
\hline B_1 & 2496 & 2532 & 4421 & 5529 & 21799 & 31046 & 57660 & Inf & 96492 & 127235 & 358538 & 407489 \\
\hline B_10 & 250 & 363 & 557 & 553 & 1875 & 2180 & 5766 & Inf & 9650 & 12729 & 35854 & 40750 \\
\hline B_20 & 127 & 217 & 133 & 302 & 575 & 1251 & 2883 & 399044 & 4825 & 6365 & 17927 & 20375 \\
\hline B_30 & 60 & 60 & 143 & 185 & 383 & 834 & 1922 & 261661 & 3217 & 4239 & 11952 & 13583 \\
\hline B_40 & 43 & 45 & 77 & 151 & 189 & 599 & 1157 & 189616 & 2422 & 3183 & 8964 & 10188 \\
\hline B_50 & 27 & 36 & 60 & 111 & 122 & 458 & 924 & 154072 & 1930 & 2546 & 7172 & 8150 \\
\hline A_1 & 280 & 1956 & 222 & 1131 & 171 & 1326 & 4133 & Inf & 10542 & 46623 & 19313 & 68970 \\
\hline A_10 & 29 & 210 & 24 & 115 & 24 & 134 & 415 & 270500 & 1106 & 4667 & 1934 & 6903 \\
\hline A_20 & 15 & 106 & 12 & 58 & 11 & 69 & 208 & 133996 & 572 & 2336 & 969 & 3455 \\
\hline A_30 & 11 & 72 & 9 & 39 & 9 & 47 & 139 & 91106 & 390 & 1559 & 647 & 2305 \\
\hline A_40 & 9 & 54 & 7 & 31 & 8 & 36 & 105 & 67898 & 293 & 1170 & 487 & 1731 \\
\hline A_50 & 8 & 44 & 5 & 25 & 7 & 9 & 92 & 54637 & 160 & 937 & 390 & 1386 \\
\hline
\end{tabular}

Algorithms ranking by needed computing time before convergence

\begin{tabular}{|c|c|c|c|c|c|c|c|c|c|c|c|c|c|}
\hline Algo & $\begin{array}{l}\text { Simu1 } \\
p=50 \\
r=3\end{array}$ & $\begin{array}{l}\text { Simu1 } \\
p=50 \\
r=5\end{array}$ & $\begin{array}{l}\text { Simu1 } \\
p=100 \\
r=3\end{array}$ & $\begin{array}{l}\text { Simu1 } \\
p=100 \\
r=5\end{array}$ & $\begin{array}{l}\text { Simu1 } \\
p=500 \\
r=3\end{array}$ & $\begin{array}{l}\text { Simu1 } \\
p=500 \\
r=5\end{array}$ & $\begin{array}{l}\text { Simu2 } \\
p=50 \\
r=3\end{array}$ & $\begin{array}{l}\text { Simu2 } \\
p=50 \\
r=5\end{array}$ & $\begin{array}{l}\text { Simu2 } \\
p=100 \\
r=3\end{array}$ & $\begin{array}{l}\text { Simu2 } \\
p=100 \\
r=5\end{array}$ & $\begin{array}{l}\text { Simu2 } \\
p=500 \\
r=3\end{array}$ & $\begin{array}{l}\text { Simu2 } \\
p=500 \\
r=5\end{array}$ & $\begin{array}{l}\text { mean } \\
\text { rank }\end{array}$ \\
\hline A_50 & 1.0 & 7.0 & 3.0 & 3.0 & 5.0 & 1.0 & 1.0 & 1.0 & 1.0 & 2.0 & 1.0 & 1.0 & 2.25 \\
\hline A_20 & 5.0 & 4.0 & 2.0 & 1.0 & 1.0 & 4.0 & 3.0 & 4.0 & 2.0 & 1.0 & 4.0 & 4.0 & 2.92 \\
\hline A_30 & 2.0 & 6.0 & 4.0 & 2.0 & 2.0 & 3.0 & 4.0 & 3.0 & 3.0 & 5.0 & 3.0 & 3.0 & 3.33 \\
\hline A_40 & 4.0 & 5.0 & 5.0 & 4.0 & 4.0 & 2.0 & 2.0 & 2.0 & 5.0 & 3.0 & 2.0 & 2.0 & 3.33 \\
\hline A_10 & 3.0 & 8.0 & 1.0 & 5.0 & 3.0 & 5.0 & 5.0 & 5.0 & 4.0 & 4.0 & 5.0 & 5.0 & 4.42 \\
\hline B_50 & 8.0 & 1.0 & 8.0 & 7.0 & 7.0 & 7.0 & 6.0 & 6.0 & 8.0 & 7.0 & 7.0 & 6.0 & 6.5 \\
\hline B_40 & 7.0 & 2.0 & 10.0 & 8.0 & 8.0 & 8.0 & 7.0 & 7.0 & 10.0 & 8.0 & 8.0 & 7.0 & 7.5 \\
\hline B_30 & 6.0 & 3.0 & 9.0 & 9.0 & 9.0 & 9.0 & 9.0 & 8.0 & 11.0 & 9.0 & 9.0 & 8.0 & 8.25 \\
\hline A_1 & 10.0 & 11.0 & 6.0 & 11.0 & 6.0 & 6.0 & 8.0 & 10.0 & 6.0 & 11.0 & 6.0 & 9.0 & 8.33 \\
\hline B_20 & 9.0 & 9.0 & 7.0 & 6.0 & 10.0 & 10.0 & 10.0 & 9.0 & 7.0 & 6.0 & 10.0 & 10.0 & 8.58 \\
\hline B_10 & 11.0 & 10.0 & 11.0 & 10.0 & 11.0 & 11.0 & 11.0 & 10.0 & 9.0 & 10.0 & 11.0 & 11.0 & 10.5 \\
\hline B_1 & 12.0 & 12.0 & 12.0 & 12.0 & 12.0 & 12.0 & 12.0 & 10.0 & 12.0 & 12.0 & 12.0 & 12.0 & 11.83 \\
\hline
\end{tabular}

Algorithms ranking by needed number of iterations before convergence

\begin{tabular}{|c|c|c|c|c|c|c|c|c|c|c|c|c|c|}
\hline Algo & $\begin{array}{l}\text { Simu1 } \\
p=50 \\
r=3\end{array}$ & $\begin{array}{l}\text { Simu1 } \\
p=50 \\
r=5\end{array}$ & $\begin{array}{l}\text { Simu1 } \\
p=100 \\
r=3\end{array}$ & $\begin{array}{l}\text { Simu1 } \\
p=100 \\
r=5\end{array}$ & $\begin{array}{l}\text { Simu1 } \\
p=500 \\
r=3\end{array}$ & $\begin{array}{l}\text { Simu1 } \\
p=500 \\
r=5\end{array}$ & $\begin{array}{l}\text { Simu2 } \\
p=50 \\
r=3\end{array}$ & $\begin{array}{l}\text { Simu2 } \\
p=50 \\
r=5\end{array}$ & $\begin{array}{l}\text { Simu2 } \\
p=100 \\
r=3\end{array}$ & $\begin{array}{l}\text { Simu2 } \\
p=100 \\
r=5\end{array}$ & $\begin{array}{l}\text { Simu2 } \\
p=500 \\
r=3\end{array}$ & $\begin{array}{l}\text { Simu2 } \\
p=500 \\
r=5\end{array}$ & $\begin{array}{l}\text { mean } \\
\text { rank }\end{array}$ \\
\hline A_50 & 1.0 & 2.0 & 1.0 & 1.0 & 1.0 & 1.0 & 1.0 & 1.0 & 1.0 & 1.0 & 1.0 & 1.0 & 1.08 \\
\hline $\begin{array}{l}\text { A_40 } \\
\end{array}$ & 2.0 & 4.0 & 2.0 & 2.0 & 2.0 & 2.0 & 2.0 & 2.0 & 2.0 & 2.0 & 2.0 & 2.0 & 2.17 \\
\hline A_30 & 3.0 & 6.0 & 3.0 & 3.0 & 3.0 & 3.0 & 3.0 & 3.0 & 3.0 & 3.0 & 3.0 & 3.0 & 3.25 \\
\hline A_20 & 4.0 & 7.0 & 4.0 & 4.0 & 4.0 & 4.0 & 4.0 & 4.0 & 4.0 & 4.0 & 4.0 & 4.0 & 4.25 \\
\hline B_50 & 5.0 & 1.0 & 6.0 & 5.0 & 6.0 & 6.0 & 6.0 & 5.0 & 6.0 & 5.0 & 6.0 & 6.0 & 5.25 \\
\hline A_10 & 6.0 & 8.0 & 5.0 & 6.0 & 5.0 & 5.0 & 5.0 & 8.0 & 5.0 & 8.0 & 5.0 & 5.0 & 5.92 \\
\hline B_40 & 7.0 & 3.0 & 7.0 & 7.0 & 8.0 & 7.0 & 7.0 & 6.0 & 7.0 & 6.0 & 7.0 & 7.0 & 6.58 \\
\hline B_30 & 8.0 & 5.0 & 9.0 & 8.0 & 9.0 & 8.0 & 8.0 & 7.0 & 8.0 & 7.0 & 8.0 & 8.0 & 7.75 \\
\hline B_20 & 9.0 & 9.0 & 8.0 & 9.0 & 10.0 & 9.0 & 9.0 & 9.0 & 9.0 & 9.0 & 9.0 & 9.0 & 9.0 \\
\hline A_1 & 11.0 & 11.0 & 10.0 & 11.0 & 7.0 & 10.0 & 10.0 & 10.0 & 11.0 & 11.0 & 10.0 & 11.0 & 10.25 \\
\hline B_10 & 10.0 & 10.0 & 11.0 & 10.0 & 11.0 & 11.0 & 11.0 & 10.0 & 10.0 & 10.0 & 11.0 & 10.0 & 10.42 \\
\hline B_1 & 12.0 & 12.0 & 12.0 & 12.0 & 12.0 & 12.0 & 12.0 & 10.0 & 12.0 & 12.0 & 12.0 & 12.0 & 11.83 \\
\hline
\end{tabular}


Fixed datasets

Datasets description

3 datasets were used: Breast_cancer, California_housing and Bio_train, all of which have continuous values:

\begin{tabular}{|c|c|c|l|}
\hline Dataset & $\begin{array}{c}\text { Number } \\
\text { of columns }\end{array}$ & $\begin{array}{c}\text { Number } \\
\text { of lines }\end{array}$ & \multicolumn{1}{|c|}{ Link } \\
\hline Breast_cancer & 8 & 569 & $\begin{array}{l}\text { https://archive.ics.uci.edu/ml/datasets/Breast+Cancer } \\
\text { +Wisconsin+\%28Diagnostic\%29 }\end{array}$ \\
\hline California_housing & 9 & 20640 & https://www.dcc.fc.up.pt/ ltorgo/Regression/DataSets.html \\
\hline Bio_train & 77 & 145751 & http://osmot.cs.cornell.edu/kddcup/datasets.html \\
\hline
\end{tabular}

The following results were obtained :

Needed computing time before convergence

\begin{tabular}{|l|l|l|l|l|l|l|}
\hline Algorithms & $\begin{array}{l}\text { breast_cancer } \\
\text { r=3 }\end{array}$ & $\begin{array}{l}\text { breast_cancer } \\
\text { r=5 }\end{array}$ & $\begin{array}{l}\text { cal_housing } \\
\mathbf{r = 3}\end{array}$ & $\begin{array}{l}\text { cal_housing } \\
\mathbf{r = 5}\end{array}$ & $\begin{array}{l}\text { bio_train } \\
\text { r=3 }\end{array}$ & $\begin{array}{l}\text { bio_train } \\
\text { r=5 }\end{array}$ \\
\hline B_1 & 20.13 & 165.91 & Inf & Inf & 92.77 & 173.3 \\
\hline B_10 & 4.94 & 19.77 & Inf & 86.69 & 14.95 & 30.59 \\
\hline B_20 & 4.3 & 13.23 & 163.83 & 268.39 & 10.45 & 23.99 \\
\hline B_30 & 2.79 & 9.57 & 159.09 & 45.25 & 9.19 & 18.24 \\
\hline B_40 & 2.01 & 8.7 & 139.1 & 162.02 & 8.44 & 16.44 \\
\hline B_50 & 2.59 & 7.68 & 28.14 & 31.85 & 13.03 & 14.56 \\
\hline A_1 & 0.24 & 1.37 & 0.48 & 0.57 & 2.23 & 3.22 \\
\hline A_10 & 0.04 & 0.19 & 0.06 & 0.07 & 0.31 & 0.48 \\
\hline A_20 & 0.03 & 0.11 & 0.07 & 0.06 & 0.28 & 0.27 \\
\hline A_30 & 0.03 & 0.09 & 0.05 & 0.02 & 0.17 & 0.27 \\
\hline A_40 & 0.02 & 0.09 & 0.03 & 0.03 & 0.05 & 0.25 \\
\hline A_50 & 0.02 & 0.08 & 0.02 & 0.14 & 0.08 & 0.23 \\
\hline
\end{tabular}

Needed number of iterations before convergence

\begin{tabular}{|l|l|l|l|l|l|l|}
\hline Algorithms & $\begin{array}{l}\text { breast_cancer } \\
\text { r=3 }\end{array}$ & $\begin{array}{l}\text { breast_cancer } \\
\text { r=5 }\end{array}$ & $\begin{array}{l}\text { cal_housing } \\
\text { r=3 }\end{array}$ & $\begin{array}{l}\text { cal_housing } \\
\text { r=5 }\end{array}$ & $\begin{array}{l}\text { bio_train } \\
\text { r=3 }\end{array}$ & $\begin{array}{l}\text { bio_train } \\
\text { r=5 }\end{array}$ \\
\hline B_1 & 49220 & 317329 & Inf & Inf & 184678 & 261092 \\
\hline B_10 & 7046 & 31734 & Inf & 148624 & 18470 & 26108 \\
\hline B_20 & 3523 & 15867 & 279499 & 336046 & 9235 & 13055 \\
\hline B_30 & 2349 & 10581 & 224031 & 49529 & 6156 & 8704 \\
\hline B_40 & 1762 & 7936 & 168026 & 168023 & 4617 & 6527 \\
\hline B_50 & 1756 & 6359 & 29720 & 29718 & 3694 & 5222 \\
\hline A_1 & 538 & 2457 & 1301 & 1262 & 4467 & 4505 \\
\hline A_10 & 55 & 248 & 134 & 130 & 448 & 452 \\
\hline A_20 & 28 & 126 & 69 & 67 & 225 & 226 \\
\hline A_30 & 20 & 85 & 49 & 21 & 150 & 151 \\
\hline A_40 & 15 & 64 & 39 & 43 & 38 & 114 \\
\hline A_50 & 12 & 53 & 39 & 72 & 31 & 91 \\
\hline
\end{tabular}


Algorithms ranking by needed computing time before convergence

\begin{tabular}{|l|l|l|l|l|l|l|l|}
\hline Algorithms & $\begin{array}{l}\text { breast_cancer } \\
\text { r=3 }\end{array}$ & $\begin{array}{l}\text { breast_cancer } \\
\text { r=5 }\end{array}$ & $\begin{array}{l}\text { cal_housing } \\
\mathbf{r = 3}\end{array}$ & $\begin{array}{l}\text { cal_housing } \\
\mathbf{r = 5}\end{array}$ & $\begin{array}{l}\text { bio_train } \\
\text { r=3 }\end{array}$ & $\begin{array}{l}\text { bio_train } \\
\text { r=5 }\end{array}$ & mean rank \\
\hline A_40 & 1.0 & 3.0 & 2.0 & 2.0 & 1.0 & 2.0 & 1.83 \\
\hline A_50 & 2.0 & 1.0 & 1.0 & 5.0 & 2.0 & 1.0 & 2.0 \\
\hline A_30 & 3.0 & 2.0 & 3.0 & 1.0 & 3.0 & 3.0 & 2.5 \\
\hline A_20 & 4.0 & 4.0 & 5.0 & 3.0 & 4.0 & 4.0 & 4.0 \\
\hline A_10 & 5.0 & 5.0 & 4.0 & 4.0 & 5.0 & 5.0 & 4.67 \\
\hline A_1 & 6.0 & 6.0 & 6.0 & 6.0 & 6.0 & 6.0 & 6.0 \\
\hline B_50 & 8.0 & 7.0 & 7.0 & 7.0 & 10.0 & 7.0 & 7.67 \\
\hline B_40 & 7.0 & 8.0 & 8.0 & 10.0 & 7.0 & 8.0 & 8.0 \\
\hline B_30 & 9.0 & 9.0 & 9.0 & 8.0 & 8.0 & 9.0 & 8.67 \\
\hline B_20 & 10.0 & 10.0 & 10.0 & 11.0 & 9.0 & 10.0 & 10.0 \\
\hline B_10 & 11.0 & 11.0 & 11.0 & 9.0 & 11.0 & 11.0 & 10.67 \\
\hline B_1 & 12.0 & 12.0 & 11.0 & 12.0 & 12.0 & 12.0 & 11.83 \\
\hline
\end{tabular}

Algorithms ranking by needed number of iterations before convergence

\begin{tabular}{|l|l|l|l|l|l|l|l|}
\hline Algorithms & $\begin{array}{l}\text { breast_cancer } \\
\mathbf{r = 3}\end{array}$ & $\begin{array}{l}\text { breast_cancer } \\
\mathbf{r = 5}\end{array}$ & $\begin{array}{l}\text { cal_housing } \\
\mathbf{r = 3}\end{array}$ & $\begin{array}{l}\text { cal_housing } \\
\mathbf{r = 5}\end{array}$ & $\begin{array}{l}\text { bio_train } \\
\mathbf{r = 3}\end{array}$ & $\begin{array}{l}\text { bio_train } \\
\text { r=5 }\end{array}$ & mean rank \\
\hline A_50 & 1.0 & 1.0 & 1.0 & 4.0 & 1.0 & 1.0 & 1.5 \\
\hline A_40 & 2.0 & 2.0 & 1.0 & 2.0 & 2.0 & 2.0 & 1.83 \\
\hline A_30 & 3.0 & 3.0 & 3.0 & 1.0 & 3.0 & 3.0 & 2.67 \\
\hline A_20 & 4.0 & 4.0 & 4.0 & 3.0 & 4.0 & 4.0 & 3.83 \\
\hline A_10 & 5.0 & 5.0 & 5.0 & 5.0 & 5.0 & 5.0 & 5.0 \\
\hline A_1 & 6.0 & 6.0 & 6.0 & 6.0 & 7.0 & 6.0 & 6.17 \\
\hline B_50 & 7.0 & 7.0 & 7.0 & 7.0 & 6.0 & 7.0 & 6.83 \\
\hline B_40 & 8.0 & 8.0 & 8.0 & 10.0 & 8.0 & 8.0 & 8.33 \\
\hline B_30 & 9.0 & 9.0 & 9.0 & 8.0 & 9.0 & 9.0 & 8.83 \\
\hline B_20 & 10.0 & 10.0 & 10.0 & 11.0 & 10.0 & 10.0 & 10.17 \\
\hline B_10 & 11.0 & 11.0 & 11.0 & 9.0 & 11.0 & 11.0 & 10.67 \\
\hline B_1 & 12.0 & 12.0 & 11.0 & 12.0 & 12.0 & 12.0 & 11.83 \\
\hline
\end{tabular}

Recap: Important rankings (computing time)

\begin{tabular}{|c|c|c|c|c|c|c|c|c|c|c|c|c|c|}
\hline Algo & $\begin{array}{l}\text { Simu1 } \\
p=50 \\
r=3\end{array}$ & $\begin{array}{l}\text { Simu1 } \\
p=50 \\
r=5\end{array}$ & $\begin{array}{l}\text { Simu1 } \\
p=100 \\
r=3\end{array}$ & $\begin{array}{l}\text { Simu1 } \\
p=100 \\
r=5\end{array}$ & $\begin{array}{l}\text { Simu1 } \\
p=500 \\
r=3\end{array}$ & $\begin{array}{l}\text { Simu1 } \\
p=500 \\
r=5\end{array}$ & $\begin{array}{l}\text { Simu2 } \\
p=50 \\
r=3\end{array}$ & $\begin{array}{l}\text { Simu2 } \\
p=50 \\
r=5\end{array}$ & $\begin{array}{l}\text { Simu2 } \\
p=100 \\
r=3\end{array}$ & $\begin{array}{l}\text { Simu2 } \\
p=100 \\
r=5\end{array}$ & $\begin{array}{l}\text { Simu2 } \\
p=500 \\
r=3\end{array}$ & $\begin{array}{l}\text { Simu2 } \\
p=500 \\
r=5\end{array}$ & $\begin{array}{l}\text { mean } \\
\text { rank }\end{array}$ \\
\hline A_50 & 1.0 & 7.0 & 3.0 & 3.0 & 5.0 & 1.0 & 1.0 & 1.0 & 1.0 & 2.0 & 1.0 & 1.0 & 2.25 \\
\hline A_20 & 5.0 & 4.0 & 2.0 & 1.0 & 1.0 & 4.0 & 3.0 & 4.0 & 2.0 & 1.0 & 4.0 & 4.0 & 2.92 \\
\hline A_30 & 2.0 & 6.0 & 4.0 & 2.0 & 2.0 & 3.0 & 4.0 & 3.0 & 3.0 & 5.0 & 3.0 & 3.0 & 3.33 \\
\hline A_40 & 4.0 & 5.0 & 5.0 & 4.0 & 4.0 & 2.0 & 2.0 & 2.0 & 5.0 & 3.0 & 2.0 & 2.0 & 3.33 \\
\hline A_10 & 3.0 & 8.0 & 1.0 & 5.0 & 3.0 & 5.0 & 5.0 & 5.0 & 4.0 & 4.0 & 5.0 & 5.0 & 4.42 \\
\hline B_50 & 8.0 & 1.0 & 8.0 & 7.0 & 7.0 & 7.0 & 6.0 & 6.0 & 8.0 & 7.0 & 7.0 & 6.0 & 6.5 \\
\hline B_40 & 7.0 & 2.0 & 10.0 & 8.0 & 8.0 & 8.0 & 7.0 & 7.0 & 10.0 & 8.0 & 8.0 & 7.0 & 7.5 \\
\hline B_30 & 6.0 & 3.0 & 9.0 & 9.0 & 9.0 & 9.0 & 9.0 & 8.0 & 11.0 & 9.0 & 9.0 & 8.0 & 8.25 \\
\hline A_1 & 10.0 & 11.0 & 6.0 & 11.0 & 6.0 & 6.0 & 8.0 & 10.0 & 6.0 & 11.0 & 6.0 & 9.0 & 8.33 \\
\hline B_20 & 9.0 & 9.0 & 7.0 & 6.0 & 10.0 & 10.0 & 10.0 & 9.0 & 7.0 & 6.0 & 10.0 & 10.0 & 8.58 \\
\hline B_10 & 11.0 & 10.0 & 11.0 & 10.0 & 11.0 & 11.0 & 11.0 & 10.0 & 9.0 & 10.0 & 11.0 & 11.0 & 10.5 \\
\hline B_1 & 12.0 & 12.0 & 12.0 & 12.0 & 12.0 & 12.0 & 12.0 & 10.0 & 12.0 & 12.0 & 12.0 & 12.0 & 11.83 \\
\hline
\end{tabular}




\begin{tabular}{|l|l|l|l|l|l|l|l|}
\hline Algorithms & $\begin{array}{l}\text { breast_cancer } \\
\text { r=3 }\end{array}$ & $\begin{array}{l}\text { breast_cancer } \\
\text { r=5 }\end{array}$ & $\begin{array}{l}\text { cal_housing } \\
\mathbf{r = 3}\end{array}$ & $\begin{array}{l}\text { cal_housing } \\
\mathbf{r = 5}\end{array}$ & $\begin{array}{l}\text { bio_train } \\
\text { r=3 }\end{array}$ & $\begin{array}{l}\text { bio_train } \\
\text { r=5 }\end{array}$ & mean rank \\
\hline A_40 & 1.0 & 3.0 & 2.0 & 2.0 & 1.0 & 2.0 & 1.83 \\
\hline A_50 & 2.0 & 1.0 & 1.0 & 5.0 & 2.0 & 1.0 & 2.0 \\
\hline A_30 & 3.0 & 2.0 & 3.0 & 1.0 & 3.0 & 3.0 & 2.5 \\
\hline A_20 & 4.0 & 4.0 & 5.0 & 3.0 & 4.0 & 4.0 & 4.0 \\
\hline A_10 & 5.0 & 5.0 & 4.0 & 4.0 & 5.0 & 5.0 & 4.67 \\
\hline A_1 & 6.0 & 6.0 & 6.0 & 6.0 & 6.0 & 6.0 & 6.0 \\
\hline B_50 & 8.0 & 7.0 & 7.0 & 7.0 & 10.0 & 7.0 & 7.67 \\
\hline B_40 & 7.0 & 8.0 & 8.0 & 10.0 & 7.0 & 8.0 & 8.0 \\
\hline B_30 & 9.0 & 9.0 & 9.0 & 8.0 & 8.0 & 9.0 & 8.67 \\
\hline B_20 & 10.0 & 10.0 & 10.0 & 11.0 & 9.0 & 10.0 & 10.0 \\
\hline B_10 & 11.0 & 11.0 & 11.0 & 9.0 & 11.0 & 11.0 & 10.67 \\
\hline B_1 & 12.0 & 12.0 & 11.0 & 12.0 & 12.0 & 12.0 & 11.83 \\
\hline
\end{tabular}

\section{References}

[1] A. Balsubramani, S. Dasgupta, Y. Freund, The fast convergence of incremental PCA, Advances in Neural Information Processing Systems 26 (2013) 3174-3182.

[2] J.P. Benzécri, Approximation stochastique dans une algèbre normée non commutative, Bulletin de la Société Mathématique de France 97 (1969) 225-241.

[3] O. Brandière, Un algorithme du gradient pour l'analyse en composantes principales, Prépublication 14, Université de Marne-la-Vallée (1994).

[4] O. Brandière, Un algorithme pour l'analyse en composantes principales, Comptes Rendus de l'Académie des Sciences 321 (série I) (1995) 233-236.

[5] O. Brandière, Some pathological traps for stochastic approximation, SIAM J. Control Optim. 36 (4) (1998) $1293-1314$.

[6] O. Brandière, M. Duflo, Les algorithmes stochastiques contournent-ils les pièges ? Annales de l'Institut Henri Poincaré, Probabilités et Statistique 32 (1996) 395-427.

[7] T. Budavari, V. Wild, a.s. Szalay, L. Dobos, C.W. Yip, Reliable eigenspectra for new generation surveys, Monthly Notices of the Royal Astronomical Society 394 (2009) 1496-1502.

[8] H. Cardot, P. Cénac, J.M. Monnez, A fast and recursive algorithm for clustering large datasets with k-medians, Computational Statistics and Data Analysis 56 (2012) 1434-1449.

[9] H. Cardot, D. Degras, Online principal component analysis in high dimension: which algorithm to choose ? International Statistical Review 86 (2018) 29-50.

[10] J.D. Carroll, A generalization of canonical correlation analysis to three or more sets of variables, Proceedings of the 76th Convention American. Psychological Association (1968) 227-228.

[11] S. Deerwester, S.T. Dumais, G.W. Furnas, T.K. Landauer, R. Harshman, Indexing by latent semantic analysis, Journal of the American Society for Information Science 41 (6) (1990) 391-407.

[12] K. Duarte, J.M. Monnez, E. Albuisson, Sequential linear regression with online standardized data, PLoS ONE, Public Library of Science (2018) 1-27, https://doi.org/10.1371/journal.pone.0191186.

[13] M. Duflo, Random Iterative Models, Applications in Mathematics, 34, Springer-Verlag, Berlin, 1997.

[14] M. Greenacre, J. Blasius, ed., Multiple Correspondence Analysis and Related Methods, Chapman \& Hall/CRC, New-York, 2006.

[15] J. Karhunen, E. Oja, New methods for stochastic approximation of truncated Karhunen-Loève expansions, Proceedings of the 6th International Conference on Pattern Recognition, IEEE Computer Society Press (1982) 550-553.

[16] T.P. Krasulina, Method of stochastic approximation in the determination of the largest eigenvalue of the mathematical expectation of random matrices, Automation and Remote Control 2 (1970) 215-221.

[17] C.D. Manning, P. Raghavan P, H. Schütze, Matrix decompositions and latent semantic indexing, Introduction to Information Retrieval, Cambridge University Press, Cambridge, 2008.

[18] A. Markos, A.I. D’Enza, Incremental generalized canonical correlation analysis, Analysis of Large and Complex Data, Wilhem AFX, Kestler HA (eds), Studies in Classification, Data Analysis and Knowledge Organization, Springer, 2016, 185-194.

[19] E. Oja, A simplified neuron model as a principal component analyser, Journal of Mathematical Biology 15 (1982) $267-273$.

[20] E. Oja E, J. Karhunen, On stochastic approximation of the eigenvectors and eigenvalues of the expectation of a random matrix, Journal of Mathematical Analysis and Applications 106 (1985) 69-84.

[21] J. Pagès, Multiple Factor Analysis by Example Using R, The R Series, Chapman \& Hall/CRC, London, 2014.

[22] H. Robbins, D. Siegmund, A convergence theorem for nonnegative almost supermartingales and some applications, Optimizing Methods in Statistics, J.S. Rustagi (ed.), Academic Press, New York, 1971, 233-257.

[23] P. Sanguansat, Principal Component Analysis-Engineering Applications, InTech, Rijeka, 2012.

[24] P. Yang, C.J. Hsieh, J.L. Wang, History PCA: a new algorithm for streaming PCA, arXiv:1802.05447v1 (2018). 